\title{
Article
}

\section{Stellar rotation rates in Kepler eccentric (heartbeat) binaries obtained from $r$ - mode signatures}

Saio, Hideyuki and Kurtz, Donald Wayne

Available at http://clok.uclan.ac.uk/40493/

Saio, Hideyuki and Kurtz, Donald Wayne ORCID: 0000-0002-1015-3268 (2022) Stellar rotation rates in Kepler eccentric (heartbeat) binaries obtained from $r$ mode signatures. Monthly Notices of the Royal Astronomical Society, 511 (1). pp. 560-571. ISSN 0035-8711

It is advisable to refer to the publisher's version if you intend to cite from the work. http://dx.doi.org/10.1093/mnras/stac048

For more information about UCLan's research in this area go to http://www.uclan.ac.uk/researchgroups/ and search for <name of research Group>.

For information about Research generally at UCLan please go to http://www.uclan.ac.uk/research/

All outputs in CLoK are protected by Intellectual Property Rights law, including Copyright law. Copyright, IPR and Moral Rights for the works on this site are retained by the individual authors and/or other copyright owners. Terms and conditions for use of this material are defined in the policies page.

\section{CLoK}

Central Lancashire online Knowledge www.clok.uclan.ac.uk

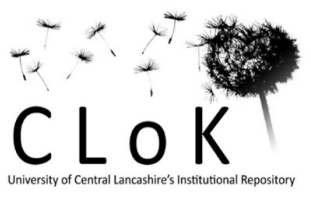




\title{
Stellar rotation rates in Kepler eccentric (heartbeat) binaries obtained from r-mode signatures
}

\author{
Hideyuki Saio ${ }^{1 \star}$ and Donald W. Kurtz ${ }^{\oplus 2,3}$ \\ ${ }^{1}$ Astronomical Institute, Graduate School of Science, Tohoku University, Sendai, Miyagi 980-8578, Japan \\ ${ }^{2}$ Centre for Space Research, Physics Department, North-West University, Mahikeng 2745, South Africa \\ ${ }^{3}$ Jeremiah Horrocks Institute, University of Central Lancashire, Preston PR1 2HE, UK
}

Accepted 2022 January 5. Received 2022 January 5; in original form 2021 November 23

\begin{abstract}
R-mode oscillations in a rotating star produce characteristic signatures in a Fourier amplitude spectrum at frequencies related with the rotation frequency, which can be, in turn, used to obtain the surface rotation rate of the star. Some binary stars observed by Kepler indicate the presence of $r$ modes that are probably excited by the tidal effect. In this paper, we have obtained stellar rotation periods in 20 eccentric (heartbeat) binaries with r-mode signatures. The majority of the rotation periods are found to be comparable to pseudo-synchronous periods, in which the angular velocity of rotation is similar to the angular orbital motion of the companion at periastron. In particular, for the heartbeat stars with orbital periods longer than about $8 \mathrm{~d}$, all but one agree with pseudo-synchronous rotation. In contrast to a previous investigation by Zimmerman et al., our result supports the pseudo-synchronisation theory developed by Hut.
\end{abstract}

Key words: binaries: eclipsing-stars: oscillations-stars: rotation.

\section{INTRODUCTION}

Among close binaries there is a subclass having large eccentricities. The light curves of such highly eccentric binary systems exhibit rapid variations caused by strong tidal effects during periastron passage. These systems are called 'heartbeat' stars (Thompson et al. 2012) because of the resemblance between the light curve and an electrocardiograph. Thanks to the precise and nearly uninterrupted observations from the Kepler satellite, the number of identified heartbeat stars has increased greatly as 172 systems are now listed in the Kepler Eclipsing Binary Catalogue (KEBC, Kirk et al. 2016; http://keplerebs.villanova.edu/). Recently, more than 200 heartbeat stars have been found in TESS data (IJspeert et al. 2021; KołaczekSzymański et al. 2021; Hambleton, private communication), and 991 in OGLE data (Wrona et al. 2021).

The presence of many eccentric binaries was predicted by Hut (1981), who found, from a theoretical analysis, that if the ratio of the orbital to rotational angular momentum is sufficiently high $(\gtrsim 7)$, the time-scale of decreasing the orbital eccentricity by tidal dissipation is much longer than the changes of other binary parameters. Hut (1981) predicted the rotation rate in such a binary system to settle into pseudo-synchronization with an eccentric orbit, in which the angular rotation velocity of the primary star should be close to (but slightly slower than) the orbital angular velocity of the secondary at the periastron (much faster than the mean motion). Such eccentric binaries should correspond to heartbeat stars.

A Fourier transform amplitude spectrum (FT hereafter) of the light curve of a heartbeat star reveals a long series of equally spaced orbital frequency harmonics caused by the strongly non-sinusoidal

^E-mail: saio@astr.tohoku.ac.jp light curve. In addition, it often shows amplitudes at frequencies associated with stellar spots and pulsations (oscillations).

Assuming that a peak (or a tight group of peaks) and harmonics separated from the orbital peaks in an FT are caused by a spot, or spots, on the rotating stellar surface, Zimmerman et al. (2017) obtained the rotation periods for stars in 24 heartbeat systems. For 18 of them with eccentricities evaluated in the literature, they compared the rotation periods obtained with pseudo-synchronous rotation periods that are determined from the orbital period and eccentricity (Hut 1981). Zimmerman et al. found that rotation periods of many heartbeat binaries are about $\frac{3}{2}$ times the pseudo-synchronous rotation periods, contrary to the prediction of Hut (1981).

Among the peaks that Zimmerman et al. (2017) adopted as rotational modulations due to surface spots, there are cases in which the signature is not a single peak but a group of peaks spread over a certain range of frequencies. Zimmerman et al. adopted the central frequency of the peak distribution as the rotation frequency, assuming the group to be caused by spots on different latitudes in the presence of latitudinal differential rotation.

Instead, we assume in the present paper such a group of frequencies to be caused by $\mathrm{r}$ modes (global Rossby waves influenced by buoyancy). The strong tidal forces would disturb the rotational flow in the stellar envelope, which would, in turn, generate Rossby waves and hence their global modes, i.e. $r$ modes, would be formed as discussed in Saio et al. (2018) and Saio (2018). The presence of $\mathrm{r}$ modes in binary stars is not limited to highly eccentric binaries like heartbeat stars, but is common in close binaries associated with strong tidal forces. For example, Saio (2020) found r-mode signatures in $\sim 700$ stars among $\sim 800$ eclipsing binary samples of the KEBC with orbital periods between 0.4 and $5 \mathrm{~d}$ and having Kepler light curves longer than three Kepler Quarters.

The r-mode features in an FT of the light curve of a star are useful to estimate the rotation rate as discussed in Saio $(2019,2020)$. 
Thus, we obtain rotation periods by fitting $\mathrm{r}$ modes with expected visibility distributions for 20 heartbeat stars having r-mode signatures selected from those analysed by Zimmerman et al. (2017) and additional heartbeat stars whose orbital eccentricities are obtained in the literature.

\section{METHOD OF ANALYSIS}

\subsection{Kepler long-cadence data to Fourier amplitude spectra}

Kepler long-cadence photometric data of heartbeat stars were downloaded from the KASOC (Kepler Asteroseismic Science Operations Center) website. ${ }^{1}$ Flux data (corrected by KASOC) for each Kepler Quarter were converted into magnitude, and the mean of the data for each Quarter was subtracted from the original data. Then, using the software PERIOD04 (Lenz \& Breger 2005) Fourier analyses were performed for the combined data for all the available Kepler Quarters for each binary system. ${ }^{2}$ We use amplitude spectra, which are referred to as FTs. All pre-whitening to produce FTs of the residuals after removing orbital harmonic series was done using PERIOD04. All of our FTs are in the low-frequency range where $r$ modes are visible. The long-cadence Kepler data have a Nyquist frequency a bit greater than $24 \mathrm{~d}^{-1}$, but we do not show the higher frequency ranges as they are not relevant to the discussion in this paper.

We explain how we proceeded with our analysis with the example of the heartbeat star KIC 8164262, on which Hambleton et al. (2018) performed detailed analysis. KIC 8164262 has a relatively long orbital period of $87.45717 \mathrm{~d}$ (from the KEBC) and a large eccentricity of 0.886 (Hambleton et al. 2018). Fig. 1 shows FTs for KIC 8164262. The upper panel shows the FT of the original light curve. The equally spaced (by $f_{\text {orb }}=0.01434 \mathrm{~d}^{-1}$ ) lines at $f_{\text {orb }}$ and many harmonics are caused by the rapid (strongly non-sinusoidal) light variation during periastron.

The orbital effects were removed by using PERIOD04 (Lenz \& Breger 2005), in which the orbital effects were expressed as a sum of jmax sinusoidal variations

$\sum_{j=1}^{j \max } A_{j} \sin \left[2 \pi\left(j f_{\text {orb }}\left(t-t_{0}\right)+\phi_{j}\right)\right]$,

with amplitude $A_{j}$ and phase $\phi_{j}$, where $t$ and $t_{0}$ are time in days and a fiducial one, respectively. Period04 determines iteratively the best set of $\left(A_{j}, \phi_{j}\right)$ for $1 \leq j \leq j \max$ to minimize the residuals. For KIC 8164262, we have included 100 harmonics $($ max $=100){ }^{3}$ The sum of the sinusoidal series was subtracted from the original data. The FT of the residual data is shown by the red line in Fig. 1. In accordance with the results of Hambleton et al. (2018), our FT shows small peaks around $0.28 \mathrm{~d}^{-1}$ as well as peaks at the rotational frequency $0.335 \mathrm{~d}^{-1}$ and its harmonic.

\subsection{R-mode frequency ranges}

Although Hambleton et al. (2018) identified the frequencies of KIC 8164262 around $0.28 \mathrm{~d}^{-1}$ as $\mathrm{g}$ modes (retrograde to the rotation), we regard these frequencies, which are lower than the rotation

\footnotetext{
${ }^{1}$ https://kasoc.phys.au.dk/

${ }^{2}$ For KIC 3230227 and KIC 11403032 , the de-trended long-cadence data were downloaded from KEBC, because lower noise levels in the FTs were obtained. ${ }^{3}$ For each case, we adopted jmax large enough that no harmonic of significant amplitude was left in the FT, or that any such harmonic was at far higher frequency than the $r$ modes so did not affect the analysis of those.
}

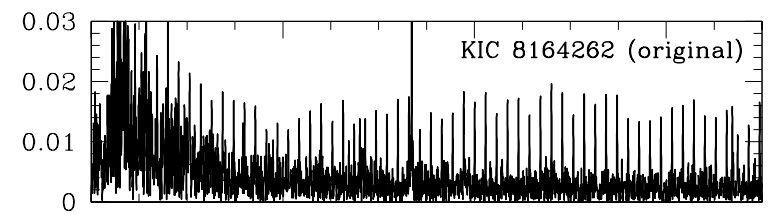

$1.70 \mathrm{M}_{\odot}, \lg ($ Teff $)=3.837, \lg \mathrm{L}=1.075, \lg \mathrm{g}=3.90, \lg \mathrm{R}=0.386$

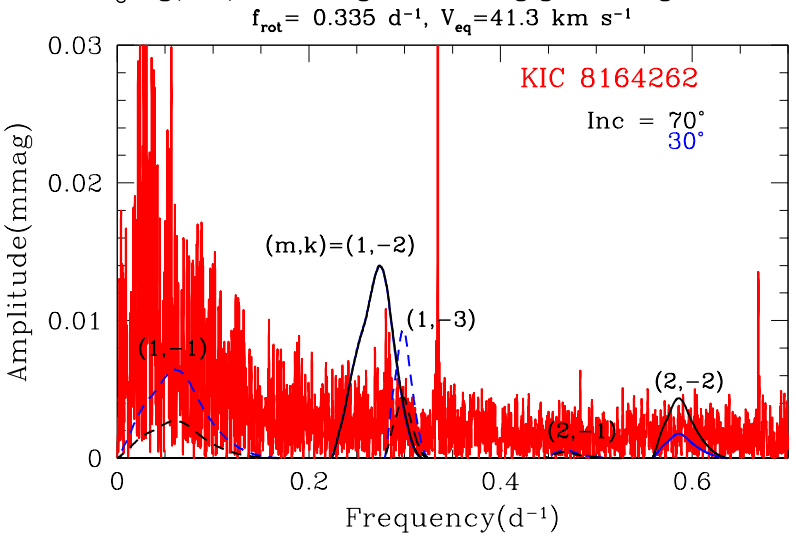

Figure 1. FTs for KIC 8164262 and r-mode visibilities at a rotation frequency of $0.335 \mathrm{~d}^{-1}$ for a $1.70-\mathrm{M}_{\odot}$ model. The upper panel shows the FT from the original light curve, while the red line in the lower panel shows the FT after the orbital harmonics have been removed. The predicted visibility distributions are shown by solid and dashed lines for even $(k=-2)$ and odd $(k=-1,-3)$ modes, respectively. Black and blue lines are for inclinations of $70^{\circ}$ and $30^{\circ}$, respectively. They are arbitrarily normalized to $0.014 \mathrm{mmag}$ at the maximum visibility for $(m, k)=(1,-2)$, for which the blue and black lines are identical. The peaks at 0.335 and $0.670 \mathrm{~d}^{-1}$ are the rotation frequency and its harmonic.

frequency, as $r$ modes. Those oscillations are global modes of Rossby waves influenced by buoyancy, whose frequency range expected in the observer's frame is given as

$m f_{\text {rot }}\left[1-\frac{2}{(m+|k|-1)(m+|k|)}\right]<v(m, k, n)<m f_{\text {rot }}$

with azimuthal order $m$ ( $>0$ for $r$ modes; in this paper, we adopt the convention that $m>0$ corresponds to retrograde modes in the co-rotating frame), latitudinal order $k(\leq-1$ for $r$ modes), and radial order $n(>0)$ (see e.g. Saio et al. 2018, for details). Even $|k|$ corresponds to a pulsation mode whose light variation on the surface is symmetric with respect to the equator, while odd $|k|$ corresponds to an antisymmetric one. The number of latitudinal nodal lines are $|k+2|$ for even $r$ modes and $|k|$ for odd $r$ modes.

In the co-rotating frame, the r-mode frequency, $v^{\mathrm{co}}(m, k, n)$, decreases with increasing radial order $n$, as for g modes. However, since the frequency in the observer's frame is given as $v(m, k$, $n)=\left|v^{\mathrm{co}}(m, k, n)-m f_{\text {rot }}\right|$ and $v^{\mathrm{co}}(m, k, n)<m f_{\text {rot }}, v(m, k, n)$ increases (getting closer to $m f_{\text {rot }}$ ) with increasing $n$. For this reason, the period spacing of $r$ modes increases with increasing period, which is opposite to the period spacings of prograde g modes, as is well known in $\gamma$ Dor stars (e.g. Van Reeth, Tkachenko \& Aerts 2016; Li et al. 2019).

If we assume that all of the $r$ modes have equal kinetic energy, we can calculate the visibility distribution as a function of pulsation frequency, by integrating the pulsation amplitude across the stellar surface (see Saio et al. 2018, for details). Examples of the visibility distribution for a model of KIC 8164262 with $f_{\text {rot }}=0.335 \mathrm{~d}^{-1}$ are shown in Fig. 1 (lower panel), in which the solid and dashed curves 
are for even $(k=-2)$ and odd $(k=-1,-3)$ modes, respectively. Unsurprisingly, odd-mode visibility is smaller for a larger inclination angle. In this figure, r-mode frequency ranges have values less than $f_{\text {rot }}$ for $m=1 \mathrm{r}$ modes, and are between $f_{\text {rot }}$ and $2 f_{\text {rot }}$ for $m=2 \mathrm{r}$ modes.

The most visible feature of $\mathrm{r}$ modes in an FT is a frequency group observed somewhere between $\frac{2}{3} f_{\text {rot }}$ and $f_{\text {rot }}$; i.e. even $\mathrm{r}$ modes of $(m$, $k)=(1,-2)$. This is a general property of $\mathrm{r}$ modes as discussed in section 3 of Saio et al. (2018). The expected visibility distribution is normalized arbitrarily at the maximum of the $(m, k)=(1,-2)$ mode visibility for all the cases in this paper. The frequency at the maximum visibility within the frequency range depends on the rotation frequency; it is close to $\frac{2}{3} f_{\text {rot }}$ for slow rotations (say $f_{\text {rot }}<$ $0.2 \mathrm{~d}^{-1}$ ), while it increases to $\sim 0.9 f_{\text {rot }}$ for $f_{\text {rot }}=0.6 \mathrm{~d}^{-1}$ in our sample. We try to fit both maximum visibility and frequency range. If it is not possible, we give priority to fit the visibility range assuming that the frequency at maximum visibility may be affected by the tidal effect which is not included.

Fig. 1 shows that the $\sim 0.28 \mathrm{~d}^{-1}$ frequencies are consistent with the visibility distribution for $r$ modes of $(m, k)=(1,-2)$ for the rotation frequency $f_{\text {rot }}=0.335 \mathrm{~d}^{-1}$, which itself agrees with the frequency at one of the peaks generated by a stellar spot. In other words, by fitting a frequency group with the visibility distribution of $r$ modes, we can obtain the rotation rate even if in the FT no peak caused by spots is present. More discussion on KIC 8164246 will be given in Section 3.1.

\subsection{Pseudo-synchronous rotation periods}

Hut (1981) found that in an eccentric binary with orbital angular momentum that is much larger than the rotational angular momentum, the orbital motion and stellar rotation attain pseudo-synchronization much faster than attaining synchronous circularization. Hut (1981) derived an analytic relation among the pseudo-synchronous rotation period $P_{\mathrm{ps}-\text { rot }}$, the orbital eccentricity $e$, and the orbital period $P_{\mathrm{orb}}$ as

$P_{\mathrm{ps}-\mathrm{rot}}=\frac{\left(1+3 e^{2}+\frac{3}{8} e^{4}\right)\left(1-e^{2}\right)^{3 / 2}}{1+\frac{15}{2} e^{2}+\frac{45}{8} e^{4}+\frac{5}{16} e^{6}} P_{\mathrm{orb}}$.

Using parameters of KIC 8164262, $e=0.886$ (Hambleton et al. 2018) and $P_{\text {orb }}=87.457 \mathrm{~d}$ in the above equation, we obtain $P_{\mathrm{ps}-\mathrm{rot}}=$ $2.976 \mathrm{~d}$, while $f_{\text {rot }}=0.335 \mathrm{~d}^{-1}$ corresponds to a rotation period of $2.985 \mathrm{~d}$ which is very close to $P_{\mathrm{ps}-\mathrm{rot}}$, indicating the system is pseudo-synchronous. We note, however, that $P_{\mathrm{ps}-\text { rot }}$ is sometimes very sensitive to the eccentricity, $e$, because equation (3) involves high order terms of $e$; for example, Zimmerman et al. (2017) obtained $P_{\text {ps-rot }}=4.22 \mathrm{~d}$ for KIC 8164262, using $e=0.857$ (Shporer et al. 2016). ${ }^{4}$

In this paper, we select 20 heartbeat binaries which show r-mode frequency groups in the FT, and whose orbital eccentricities are obtained in the literature. Comparing these r-mode features with expected $\mathrm{r}$-mode frequency ranges for assumed rotation rates we determine the rotation frequency of the primary star and compare it with the corresponding $P_{\text {ps-rot }}$. Model parameters for each case are selected mainly referring to the parameters given in Berger et al. (2020); we have chosen a mass taking into account the luminosity, then chosen an evolutionary stage which is consistent with the effective temperature. Model parameters need not be very accurate because the predicted visibility distribution of $\mathrm{r}$ modes for

${ }^{4}$ In this paper, we adopt $e=0.886$ obtained by Hambleton et al. (2018) for KIC 8164262 rather than $e=0.857$ obtained by Shporer et al. (2016), because more radial-velocity measurements were incorporated in the former analysis. a given rotation frequency is only weakly dependent on the stellar parameters, as is the rotation rate derived by fitting. Stellar models were obtained, as in our previous models for $\gamma$ Dor stars (Saio et al. 2021), using the MESA code (ver.7184 Paxton et al. 2011, 2013, 2015) adopting the same settings. In our present paper, we adopt models with diffusive overshooting of $h_{\mathrm{os}}=0.01$, in which mixing at distance $z$ from the convective-core boundary is proportional to $\exp \left[-2 z /\left(h_{\mathrm{os}} H_{\mathrm{p}}\right)\right]$ with pressure scale height $H_{\mathrm{p}}$.

\section{R-MODE FITS FOR SOME HEARTBEAT STARS}

In this section, we show the fitting of frequency groups with r-mode visibility for four selected cases, while the rest are shown in the Appendix. Fitting results and model parameters are summarized in Table 1.

\subsection{KIC 8164262}

Hambleton et al. (2018) carried out a detailed analysis of the heartbeat star KIC 8164262 to obtain the binary orbital parameters as well as stellar parameters of the primary and secondary components. They found many tidally excited oscillations whose frequencies are multiples of the orbital frequency. They also found a low frequency, $0.335 \mathrm{~d}^{-1}$, with its harmonic, which they identified as the rotation frequency, and three low-amplitude frequencies around $0.28 \mathrm{~d}^{-1}$, which they regarded as retrograde g modes.

Adopting $0.335 \mathrm{~d}^{-1}$ as the rotation frequency for a $1.70-\mathrm{M}_{\odot}$ model with $\left(\log T_{\text {eff }}, \log R\right)=(3.837,0.386)$, which are consistent with the parameters obtained by Hambleton et al. (2018), we obtained rmode visibility for $m=1$ and 2 (Fig. 1). The three frequencies found by Hambleton et al. (2018) around $0.28 \mathrm{~d}^{-1}$ are located within the frequency range of $\mathrm{r}$ modes with $(m, k)=(1,-2)$.

Although not obvious in this figure, a small hump caused by $\mathrm{r}$ modes with $(m, k)=(2,-2)$ is visible around $0.6 \mathrm{~d}^{-1}$ in fig. 7 of Hambleton et al. (2018), which confirms the r-mode interpretation for the humps in KIC 8164262. As mentioned above, the eccentricity $0.866 \pm 0.003$ obtained by Hambleton et al. (2018) yields a pseudosynchronous rotation period of $2.98 \pm 0.12 \mathrm{~d}$, which is consistent with the rotation period $2.985 \mathrm{~d}$. The rotation period with the radius of the model corresponds to an equatorial rotation velocity of $41 \mathrm{~km} \mathrm{~s}^{-1}$. Combining this velocity with $v \sin i=23 \pm 1 \mathrm{~km} \mathrm{~s}^{-1}$ obtained by Hambleton et al. (2018) yields an inclination angle of $34^{\circ}$ between the rotation axis and the line of sight, which is, in turn, inclined to the orbital axis by about $30^{\circ}$ (Hambleton et al. 2018). The misalignment between the rotation and the orbital axes is possible in the binary evolution theory of Hut (1981), in which the time-scale of the alignment is comparable to or slightly longer than the time-scale of the pseudo-synchronization (see fig. 4 of Hut 1981).

\subsection{KIC 9899216}

The orbital period of the heartbeat binary KIC 9899216 is $10.916 \mathrm{~d}$ (KEBC). Fig. 2 shows FTs for KIC 9899216 . The FT derived from the original data (upper panel) is dominated by features caused by light variations associated with the orbital motion. After pre-whitening 40 orbital harmonics from the original data, we obtain the FT of the residual data that is shown by the red line in the lower panel of Fig. 2; a clear frequency group has emerged around $0.55 \mathrm{~d}^{-1}\left(\approx 6 / P_{\text {orb }}\right)$. Its frequency range is fitted with $\mathrm{r}$ modes of $(m, k)=(1,-2)$ at a rotation frequency of $0.60 \mathrm{~d}^{-1}\left(P_{\text {rot }}=1.67 \mathrm{~d}\right)$. This is the fastest rotation among our sample of heartbeat stars. 
Table 1. Observational and model parameters, and obtained rotation periods. The orbital period $P_{\text {orb }}$ of each binary is taken from the KEBC (Kirk et al. 2016, http://keplerEBs.villanova.edu). The values in parentheses denote the uncertainty of the last digits.

\begin{tabular}{|c|c|c|c|c|c|c|c|c|c|c|c|c|}
\hline \multirow[b]{2}{*}{ KIC } & \multicolumn{3}{|c|}{ Berger et al. (2020) } & \multirow[b]{2}{*}{$\begin{array}{l}P_{\text {orb }} \\
\text { (d) }\end{array}$} & \multirow[b]{2}{*}{ eccentricity } & \multirow[b]{2}{*}{$\begin{array}{l}P_{\text {ps-rot }} \\
\text { (d) }\end{array}$} & \multicolumn{3}{|c|}{ Model parameters } & \multirow[b]{2}{*}{$\begin{array}{l}P_{\text {rot }} \\
\text { (d) }\end{array}$} & \multirow[b]{2}{*}{$\begin{array}{c}V_{\mathrm{eq}} \\
\left(\mathrm{km} \mathrm{s}^{-1}\right)\end{array}$} & \multirow[b]{2}{*}{$\operatorname{Ref}^{a}$} \\
\hline & $\begin{array}{c}T_{\text {eff }} \\
\left(10^{3} \mathrm{~K}\right)\end{array}$ & $\begin{array}{l}\lg g \\
(\mathrm{cgs})\end{array}$ & $\begin{array}{l}\lg L \\
\left(L_{\odot}\right)\end{array}$ & & & & $\begin{array}{c}M \\
\left(\mathrm{M}_{\odot}\right)\end{array}$ & $\begin{array}{c}\lg T_{\text {eff }} \\
(\mathrm{K})\end{array}$ & $\begin{array}{l}\lg L \\
\left(L_{\odot}\right)\end{array}$ & & & \\
\hline 4372379 & $6.73(14)$ & $4.23(3)$ & $0.61(54)$ & 4.5352 & $0.342(92)$ & $2.61(64)$ & 1.40 & 3.828 & 0.678 & $4.76(24)$ & 17 & $\mathrm{t} 12$ \\
\hline 5017127 & $6.29(14)$ & $4.30(11)$ & $0.36(14)$ & 20.006 & $0.550(5)$ & $5.97(11)$ & 1.20 & 3.794 & 0.442 & $6.06(19)$ & 12 & s16 \\
\hline 5034333 & $9.39(39)$ & $4.07(4)$ & $1.57(75)$ & 6.9323 & $0.5822(9)$ & $1.827(6)$ & 2.30 & 3.973 & 1.574 & $3.33(11)$ & 35 & g20 \\
\hline 5090937 & $8.21(22)$ & $3.83(3)$ & $1.49(50)$ & 8.8007 & $0.241(13)$ & $6.50(19)$ & 2.10 & 3.909 & 1.455 & $6.25(20)$ & 22 & s16 \\
\hline 5960989 & $6.48(13)$ & $3.78(3)$ & $1.10(6)$ & 50.722 & $0.810(16)$ & $3.80(50)$ & 1.70 & 3.812 & 1.086 & $4.54(22)$ & 31 & $\mathrm{~s} 16, \mathrm{~d} 17$ \\
\hline 6117415 & $6.40(13)$ & 4.04(3) & $0.70(48)$ & 19.742 & $0.7343(4)$ & $2.502(6)$ & 1.45 & 3.819 & 0.777 & $2.33(6)$ & 41 & c20 \\
\hline 8027591 & $6.10(13)$ & $3.85(6)$ & $0.79(40)$ & 24.274 & $0.586(8)$ & $6.30(20)$ & 1.50 & 3.800 & 0.859 & $6.25(20)$ & 18 & s16 \\
\hline 8164262 & $7.45(16)$ & $4.16(2)$ & $0.98(11)$ & 87.457 & $0.886(3)$ & $2.98(12)$ & 1.70 & 3.837 & 1.075 & 2.985 & 41 & h18 \\
\hline 8719324 & $7.28(17)$ & $3.96(3)$ & $1.13(17)$ & 10.233 & $0.5998(5)$ & $2.512(1)$ & 1.80 & 3.867 & 1.173 & $2.63(4)$ & 46 & g20 \\
\hline 9790355 & $6.42(13)$ & $3.79(4)$ & $1.06(12)$ & 14.566 & $0.513(7)$ & $4.96(12)$ & 1.70 & 3.812 & 1.086 & $4.54(22)$ & 31 & $\mathrm{t} 12$ \\
\hline 9899216 & $7.30(17)$ & $3.63(3)$ & $1.56(55)$ & 10.916 & $0.65(1)$ & $2.15(10)$ & 2.20 & 3.883 & 1.559 & $1.67(3)$ & 104 & $\mathrm{t} 12, \mathrm{~s} 15$ \\
\hline 11649962 & $6.80(15)$ & $4.06(3)$ & $0.81(33)$ & 10.563 & $0.5206(35)$ & $3.51(4)$ & 1.50 & 3.831 & 0.830 & $3.03(10)$ & 32 & s16 \\
\hline 11923629 & $6.23(13)$ & 4.11(4) & $0.56(62)$ & 17.973 & $0.3629(59)$ & $9.77(16)$ & 1.50 & 3.801 & 0.858 & $10.0(10)$ & 11 & s16 \\
\hline 12255108 & $7.72(18)$ & $4.05(3)$ & $1.20(18)$ & 9.1315 & $0.296(16)$ & $5.92(24)$ & 1.90 & 3.901 & 1.253 & $5.88(18)$ & 19 & s16 \\
\hline
\end{tabular}

${ }^{a}$ Sources of eccentricity: t12 = Thompson et al. (2012), s15 = Smullen \& Kobulnicky (2015), s16 = Shporer et al. (2016), d17 = Dimitrov et al. (2017), g17 = Guo et al. (2017), h18 = Hambleton et al. (2018), g20 = Guo et al. (2020), and c20 = Cheng et al. (2020).

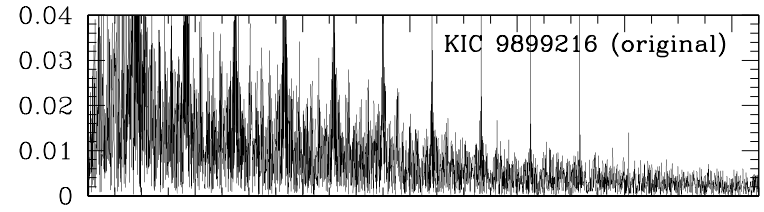

$2.20 \mathrm{M}_{\odot}, \lg ($ Teff $)=3.883, \lg \mathrm{L}=1.559, \lg \mathrm{g}=3.71, \lg \mathrm{R}=0.537$

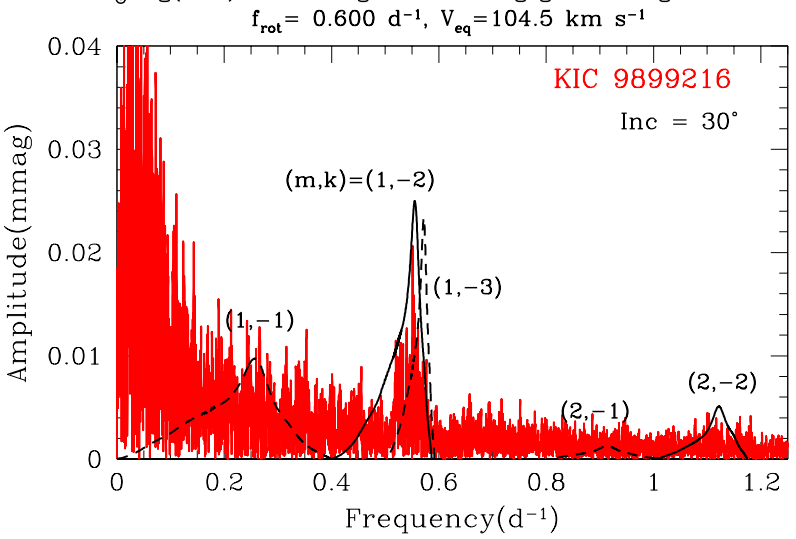

Figure 2. FTs for KIC 9899216 shown in the same format as in Fig. 1. Rmode visibilities for a low-inclination case are shown because Thompson et al. (2012) obtained an orbital inclination of $20.7 \pm 0.3$, while Smullen \& Kobulnicky (2015) estimated $6^{\circ}-15^{\circ}$.

The orbital eccentricity of KIC 9899216 was determined from the Kepler light curve data by Thompson et al. (2012), who obtained $e=0.647 \pm 0.004$, while Smullen \& Kobulnicky (2015) obtained $e=0.66 \pm 0.24$ analysing their spectroscopic radial-velocity measurements. In Table 1 , we have adopted $e=0.65 \pm 0.01$. Using the eccentricity and the orbital period $10.916 \mathrm{~d}$ in equation (3), we obtain $P_{\mathrm{ps}-\text { rot }}=2.15 \pm 0.10 \mathrm{~d}$, which is slightly larger than the rotation period obtained above.

\subsection{KIC 3230227 (HD 181850)}

KIC 3230227 is a heartbeat binary with an orbital period of $7.0471 \mathrm{~d}$. A very narrow eclipse is seen in the folded Kepler light curve (e.g. KEBC, Kirk et al. 2016). Recent orbital analyses by three groups for KIC 3230227 using the Kepler light curve and spectroscopic velocity observations have yielded similar results; Dimitrov, Kjurkchieva \& Iliev (2017) obtained an orbital eccentricity of $e=0.603 \pm 0.001$ and an orbital inclination $i=72.8 \pm 0.2$, Guo, Gies \& Fuller (2017) obtained $e=0.600 \pm 0.005, i=73.4 \pm 0.3^{\circ}$, and Smullen \& Kobulnicky (2015) obtained $e=0.60 \pm 0.04, i=66^{\circ}-71^{\circ}$ but with a slightly larger orbital period $7.051 \mathrm{~d}$ in the last case. Adopting $e=$ $0.602 \pm 0.003$ and $P_{\text {orb }}=7.0471 \mathrm{~d}$ in equation (3) yields $P_{\mathrm{ps} \text {-rot }}=$ $1.71 \pm 0.02 \mathrm{~d}$ as listed in Table 1 .

Fig. 3 shows FTs for KIC 3230227; one for the original data (upper panel) and one for the residuals after removing the orbital harmonics (lower panel). An r-mode visibility distribution for a $2.30-\mathrm{M}_{\odot}$ model at a rotation frequency of $0.23 \mathrm{~d}^{-1}$ is shown in a similar way to Fig. 1. This star has the main group of peaks at $\sim 0.18 \mathrm{~d}^{-1}$ and, as for many other cases, a secondary group of amplitudes that is formed by harmonics and combination frequencies among the main group of frequencies, which are shown by cyan lines in Fig. 3. Ignoring the secondary group, we consider only the main group. As shown in Fig. 3, the main frequency group around $0.18 \mathrm{~d}^{-1}$ is fitted with $(m, k)=(1,-2) \mathrm{r}$ modes at a rotation frequency of $0.23 \mathrm{~d}^{-1}$. The rotation frequency corresponds to $P_{\text {rot }}=4.35 \mathrm{~d}$, which is larger than the $P_{\text {ps-rot }}$ obtained above for KIC 3230227 . This deviation from the pseudo-synchronous rotation tends to occur in systems with orbital periods shorter than $8 \mathrm{~d}$ (see Table 1 and Fig. 6).

The model shown in Fig. 3 has an equatorial rotational velocity of $31 \mathrm{~km} \mathrm{~s}^{-1}$, which agrees with $v \sin i \approx 30 \mathrm{~km} \mathrm{~s}^{-1}$ obtained for 


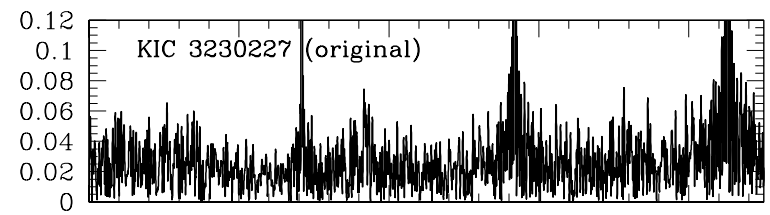

$2.30 \mathrm{M}_{\odot}, \lg ($ Teff $)=3.951, \lg \mathrm{L}=1.607, \lg \mathrm{g}=3.95, \lg \mathrm{R}=0.426$

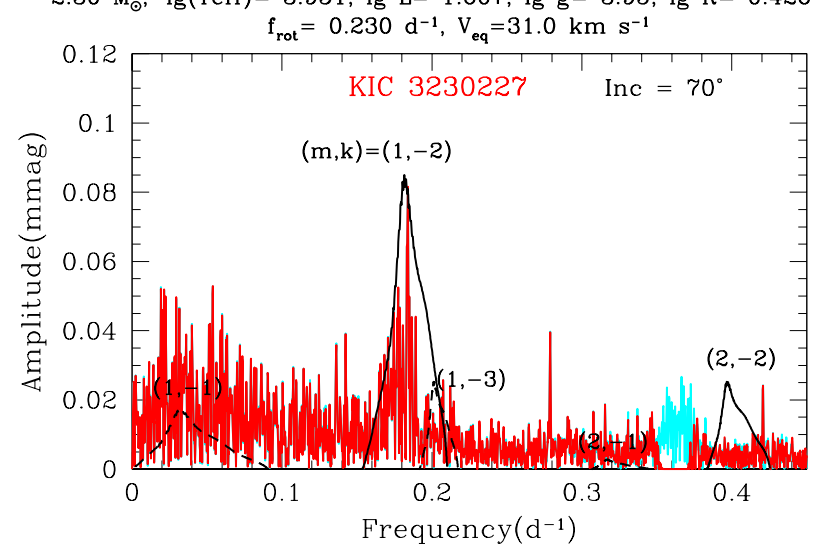

Figure 3. FTs for KIC 3230227 and theoretical visibility distributions of a model at a rotation frequency of $0.23 \mathrm{~d}^{-1}$. The upper panel shows the FT for the original light curve including orbital effects. Removing 50 orbital harmonics, we obtain the red and cyan lines in the lower panel. The cyan line indicates frequencies corresponding to harmonics and combination frequencies of peaks around $0.18 \mathrm{~d}^{-1}(>0.04 \mathrm{mmag})$. This indicates the secondary 'hump' at $\sim 0.36 \mathrm{~d}^{-1}$ need not to be considered as $m=2 \mathrm{r}$ modes.

the primary star of KIC 3230227 by Smullen \& Kobulnicky (2015) ( $\approx 75 \mathrm{~km} \mathrm{~s}^{-1}$ for the secondary).

\subsection{KIC 5017127}

The orbital period of KIC 5017127 is $20.006 \mathrm{~d}$ (KEBC) with the eccentricity $0.550 \pm 0.005$ (Shporer et al. 2016), from which we obtain $P_{\text {ps-rot }}=5.97 \pm 0.05$ d (equation 3). An FT of KIC 5017127 (Fig. 4) shows a narrow group of peaks at a frequency of $0.11 \mathrm{~d}^{-1}$, which Zimmerman et al. (2017) identified as the rotation frequency of the primary and obtained $P_{\text {rot }}=9.34 \pm 0.11 \mathrm{~d}$. Instead, we fit the frequency group with $(m, k)=(1,-2) \mathrm{r}$ modes at a rotation frequency of $0.165 \mathrm{~d}^{-1}\left(P_{\text {rot }}=6.06 \mathrm{~d}\right)$ (Fig. 4). The rotation period obtained is similar to the pseudo-synchronous rotation period, while it is smaller than the result of Zimmerman et al. (2017) by a factor of $\sim 1.5$.

\section{SUMMARY OF THE RESULTS}

The results for each heartbeat system and the adopted model parameters are summarized in Table 1 . The obtained rotation periods are compared with the pseudo-synchronous rotation periods in Fig. 5. This figure shows that for the majority of the heartbeat systems in our samples, the rotation periods of primary stars are comparable to the corresponding pseudo-synchronous rotation, confirming the theory developed by Hut (1981). [The rotation periods of KIC 11403032 and KIC 4372379 are comparable to the corresponding orbital periods (see Fig. 6)].

Our result is, however, different from the result of Zimmerman et al. (2017), which concluded that the rotation periods tend to be approximately $\frac{3}{2}$ times the corresponding pseudo-synchronous
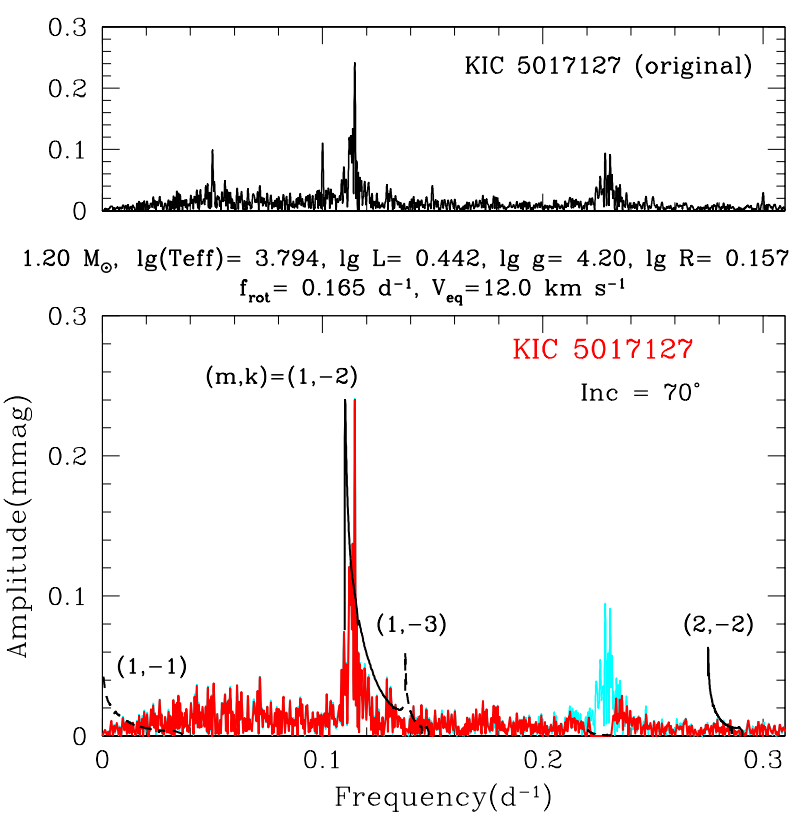

Figure 4. FTs for KIC 5017127. The upper panel shows an FT of the original light curve including the orbital harmonics from the non-sinusoidal light curve. The lower panel shows the FT after pre-whitening the orbital harmonics. The cyan line denotes harmonics and combination frequencies of the six highest amplitude $(>0.07 \mathrm{mmag}$ ) peaks in the group at $\sim 0.11 \mathrm{~d}^{-1}$. The main group is fitted with the visibility distribution of $(\mathrm{m}$, $k)=(1,-2) \mathrm{r}$ modes in a $1.2-\mathrm{M}_{\odot}$ model with a rotation frequency of $0.165 \mathrm{~d}^{-1}$. The predicted frequency range for the low mass and slow rotation model is very narrow, which is consistent with the observed amplitude group.

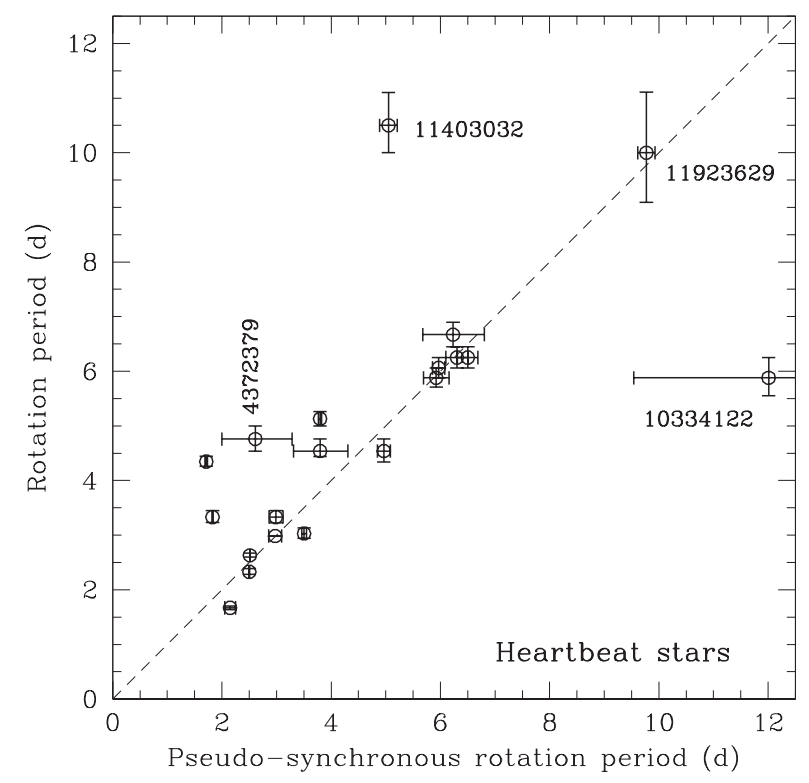

Figure 5. Rotation period obtained by r-mode fitting versus pseudosynchronous rotation period. 

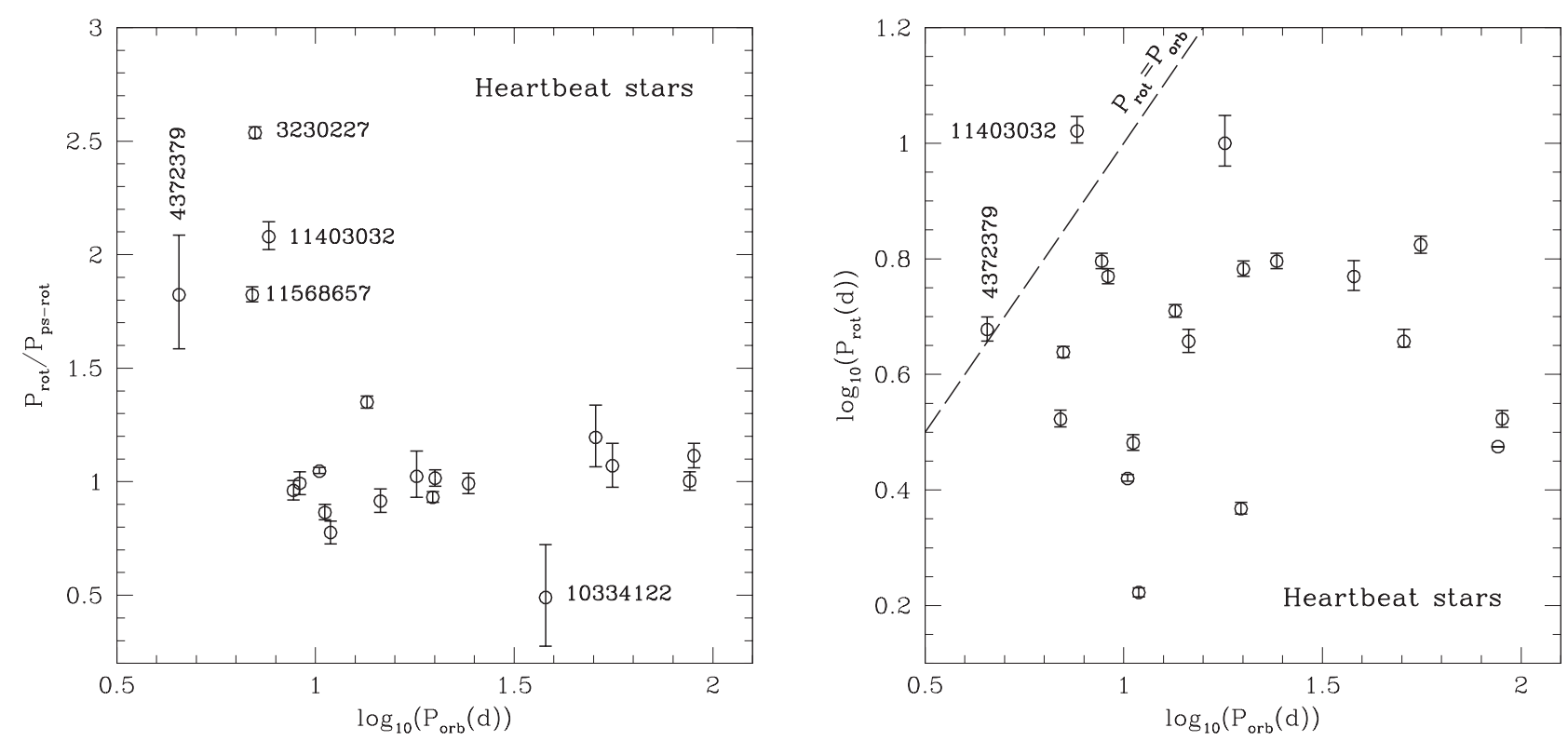

Figure 6. The left-hand panel shows the ratio of rotation period to the pseudo-synchronous rotation period versus the orbital period, while the right-hand panel shows the rotation period versus the orbital period.

rotation periods. The difference comes from Zimmerman et al. (2017) assuming that the centre of the main frequency group in the Fourier spectrum of a star is the rotation frequency, while we fit the group with $(m, k)=(1,-2) \mathrm{r}$ modes assuming a rotation frequency larger than the frequency of the main frequency group. Thus, the rotation frequency (or period) obtained by Zimmerman et al. (2017) is systematically lower (or longer) than our value which is, in most cases, consistent with pseudo-synchronous rotation. More specifically, as discussed in Section 2.2, the frequency range of the $(m, k)=(1,-2) \mathrm{r}$ modes in the observer's frame is between $\frac{2}{3} f_{\text {rot }}$ and $f_{\text {rot }}$; or the period range is between $\frac{3}{2} P_{\text {rot }}$ and $P_{\text {rot }}$.

The left-hand panel of Fig. 6 shows that in the heartbeat stars with orbital periods longer than $8 \mathrm{~d}$ pseudo-synchronization is roughly attained in all but KIC 10334122 (with large error bars), while all four systems of our sample with $P_{\text {orb }} \lesssim 8 \mathrm{~d}$ have rotation periods longer than the corresponding $P_{\text {ps-rot }}$. Among the four systems with shorter orbital periods, KIC 4372379 and KIC 11403032, which have small eccentricities of 0.342 and 0.288 , respectively, have rotation periods comparable to the orbital periods (see the right-hand panel of Fig. 6); in particular, the rotation of KIC 4372379 is almost synchronous with the orbital motion. The rotation periods of the other heartbeat stars in this figure are smaller than the corresponding orbital periods, which is because the angular velocity of the pseudo-synchronous rotation is comparable to the orbital angular velocity at periastron (Hut 1981). It is not clear to us why pseudo-synchronization does not occur in the relatively short-period systems with the orbital periods shorter than $8 \mathrm{~d}$.

\section{CONCLUSIONS}

Among the heartbeat stars observed by the Kepler satellite and collected by Kirk et al. (2016), we have selected 20 stars whose FTs show densely gathered groups of frequencies that are consistent with the visibility distribution of $\mathrm{r}$ modes, which have frequency ranges that depend on the rotation rate. For each star, we have obtained a rotation frequency by fitting the visibility distribution of $(m, k)=(1,-2) \mathrm{r}$ modes in a model whose parameters are consistent with these given by Berger et al. (2020). The rotation period thus obtained is, in most cases, found to be consistent with the pseudo-synchronous rotation supporting the orbital evolution theory developed by Hut (1981). Scrutinizing in more detail, we found that the rotation periods of all but one (KIC 10334122 with large error bars) heartbeat binaries in our samples with $P_{\text {orb }}>8 \mathrm{~d}$ are close to the pseudo-synchronous rotation period. Conversely, all four heartbeat stars with $P_{\text {orb }}<8$ d have rotation periods considerably larger than the pseudo-synchronous period. It is not clear at present the reason why the critical orbital period occurs at $8 \mathrm{~d}$ for the pseudosynchronization of heartbeat stars. This problem of understanding the angular momentum transfer rate versus orbital period is particularly interesting, given that the rotation rates in these shorter orbital period systems are sub-synchronous, so angular momentum transfer from the orbit to rotation must continue for the system to reach pseudosynchronisation.

\section{DATA AVAILABILITY}

The data underlying this article will be shared on reasonable request to the corresponding author.

\section{REFERENCES}

Berger T. A., Huber D., van Saders J. L., Gaidos E., Tayar J., Kraus A. L., 2020, AJ, 159, 280

Cheng S. J., Fuller J., Guo Z., Lehman H., Hambleton K., 2020, ApJ, 903, 122

Dimitrov D. P., Kjurkchieva D. P., Iliev I. K., 2017, MNRAS, 469, 2089

Guo Z., Gies D. R., Fuller J., 2017, ApJ, 834, 59

Guo Z., Shporer A., Hambleton K., Isaacson H., 2020, ApJ, 888, 95

Hambleton K. et al., 2018, MNRAS, 473, 5165

Hut P., 1981, A\&A, 99, 126

IJspeert L. W., Tkachenko A., Johnston C., Garcia S., De Ridder J., Van Reeth T., Aerts C., 2021, A\&A, 652, A120

Kirk B. et al., 2016, AJ, 151, 68 
Kołaczek-Szymański P. A., Pigulski A., Michalska G., Moździerski D., RóżańskiT., 2021, A\&A, 647, A12

Kurtz D. W., Shibahashi H., Murphy S. J., Bedding T. R., Bowman D. M., 2015, MNRAS, 450, 3015

Lee U., 2021, MNRAS, 505, 1495

Lee U., Saio H., 2020, MNRAS, 497, 4117

Lenz P., Breger M., 2005, Commun. Asteroseismology, 146, 53

Li G., Van Reeth T., Bedding T. R., Murphy S. J., Antoci V., 2019, MNRAS, 487, 782

Paxton B., Bildsten L., Dotter A., Herwig F., Lesaffre P., Timmes F., 2011, ApJS, 192, 3

Paxton B. et al., 2013, ApJS, 208, 4

Paxton B. et al., 2015, ApJS, 220, 15

Saio H., 2018, preprint (arXiv:1812.01253)

Saio H., 2019, MNRAS, 487, 2177

Saio H., 2020, in Neiner C., Weiss W. W., Baade D., Griffin R. E., Lovekin C. C., Moffat A. F. J., eds, Stars and their Variability Observed from Space. University of Vienna, Vienna, p. 321

Saio H., Kurtz D. W., Murphy S. J., Antoci V. L., Lee U., 2018, MNRAS, 474, 2774

Saio H., Takata M., Lee U., Li G., Van Reeth T., 2021, MNRAS, 502, 5856

Shporer A. et al., 2016, ApJ, 829, 34

Smullen R. A., Kobulnicky H. A., 2015, ApJ, 808, 166

Thompson S. E. et al., 2012, ApJ, 753, 86

Van Reeth T., Tkachenko A., Aerts C., 2016, A\&A, 593, A120

Wrona M. et al., 2021, preprint (arXiv:2109.14616)

Zimmerman M. K., Thompson S. E., Mullally F., Fuller J., Shporer A., Hambleton K., 2017, ApJ, 846, 147

\section{APPENDIX A: OTHER HEARTBEAT STARS FITTED WITH R MODES}

\section{A1 KIC 4372379}

KIC 4372379 is a short period $\left(P_{\text {orb }}=4.535 \mathrm{~d}\right.$; KEBC) heartbeat binary with an orbital eccentricity $0.342 \pm 0.092$ (Thompson et al. 2012). From equation (3), we obtain $P_{\text {ps-rot }}=2.61 \pm 0.64$ d. Fig. A1 (lower panel) shows an FT for KIC 4372379. Also shown are visibility distributions of $\mathrm{r}$ modes predicted for a $1.40-\mathrm{M}_{\odot}$ model at a rotation frequency of $0.21 \mathrm{~d}^{-1}\left(P_{\text {rot }}=4.76 \mathrm{~d}\right)$. The rotation frequency has been chosen to reproduce the frequency group around $0.14-0.18 \mathrm{~d}^{-1}$ by the expected frequency ranges of $(m, k)=(1,-2)$ and $(1,-3) \mathrm{r}$ modes. The rotation period is longer than $P_{\mathrm{ps}-\mathrm{rot}}$, while close to the orbital period (see Fig. 6).

\section{A2 KIC 5034333}

The orbital period of KIC 5034333 is $6.932 \mathrm{~d}$ (KEBC), while Guo et al. (2020) obtained an orbital eccentricity of $0.5822 \pm 0.0009 .{ }^{5}$ Using these parameters in equation (3) yields $P_{\mathrm{ps}-\mathrm{rot}}=1.827 \pm 0.006 \mathrm{~d}$. Fig. A2 (lower panel) shows an FT for KIC 5034333 with r-mode visibility distributions predicted for a $2.30-\mathrm{M}_{\odot}$ model at a rotation frequency of $0.30 \mathrm{~d}^{-1}\left(P_{\text {rot }}=3.33 \mathrm{~d}\right)$. The rotation frequency is chosen to fit the frequency range of the group at $\sim 0.25 \mathrm{~d}^{-1}$ with $\mathrm{r}$ modes of $(m, k)=(1,-2)$, while Zimmerman et al. (2017) obtained a slightly slower rotation frequency of $0.251 \mathrm{~d}^{-1}$, considering the strongest peak at that frequency to be caused by a spot on the stellar surface.

\footnotetext{
${ }^{5}$ While Thompson et al. (2012) obtained $e=0.575 \pm 0.002$ using a similar method applied to the Kepler data between Quarters 0 and 7, we have adopted in Table 1 the result of Guo et al. (2020) based on a longer Kepler data set from Quarters 0 to 17.
}

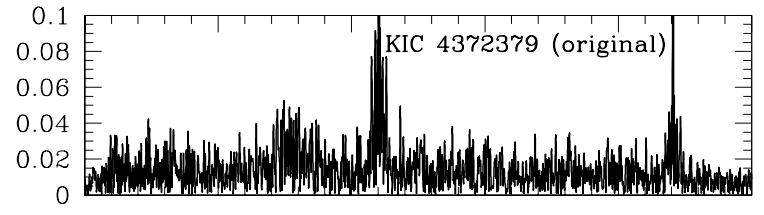

$1.40 \mathrm{M}_{\odot}, \lg (\mathrm{Teff})=3.828, \lg \mathrm{L}=0.678, \lg \mathrm{g}=4.17, \lg \mathrm{R}=0.206$

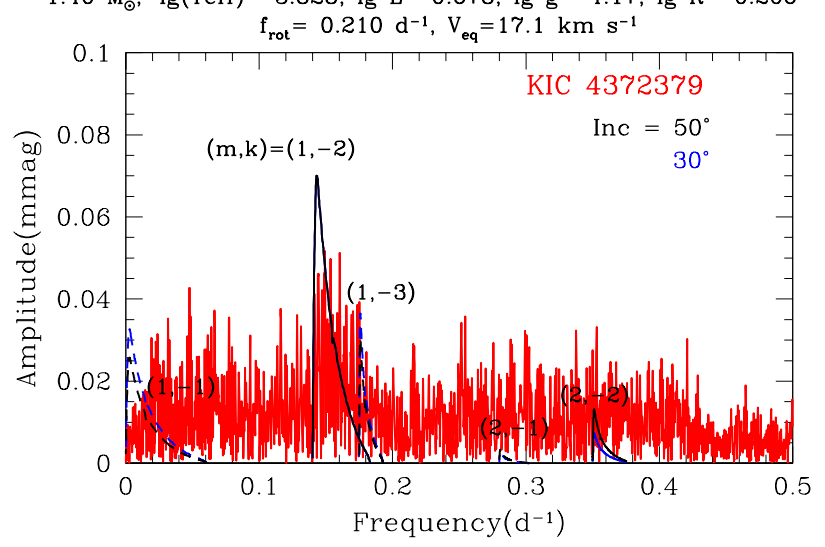

Figure A1. FTs for KIC 4372379 in the same format as Fig. 1. Thompson et al. (2012) obtained the orbital inclination to be $48^{\circ} \pm 11^{\circ}$ for this star.

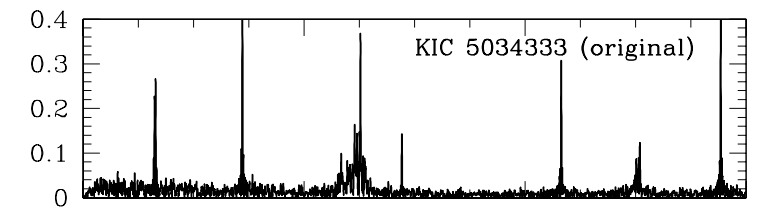

$2.30 \mathrm{M}_{\odot}, \lg ($ Teff $)=3.973, \lg \mathrm{L}=1.574, \lg \mathrm{g}=4.07, \lg \mathrm{R}=0.365$

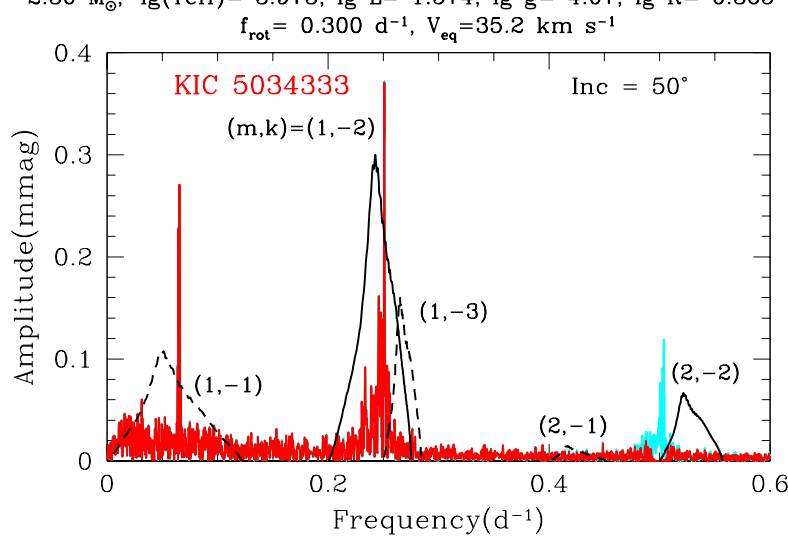

Figure A2. FTs for KIC 5034333 and r-mode visibility distributions for the indicated parameters. The cyan line denotes harmonics and combination frequencies of high-amplitude ( $>8 \mathrm{mmag}$ ) peaks at $\sim 0.25 \mathrm{~d}^{-1}$. We note that Guo et al. (2020) obtained an orbital inclination of 49.88 .

\section{A3 KIC 5090937}

KIC 5090937 is a heartbeat star with a relatively short orbital period of $8.8007 \mathrm{~d}$ (KEBC) and a relatively small orbital eccentricity of 0.241 (Shporer et al. 2016). Using these orbital parameters in equation (3) yields $P_{\mathrm{ps}-\text { rot }}=6.50 \mathrm{~d}$. Fig. A3 (lower panel) shows an FT for KIC 5090937 with r-mode visibility distribution for a $2.10-\mathrm{M}_{\odot}$ model. The frequency group at $\sim 0.12 \mathrm{~d}^{-1}$ is fitted with 


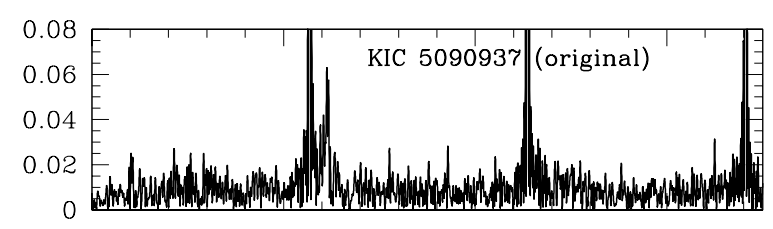

$2.10 \mathrm{M}_{\odot}, \lg (\mathrm{Teff})=3.909, \lg \mathrm{L}=1.455, \lg \mathrm{g}=3.89, \lg \mathrm{R}=0.433$

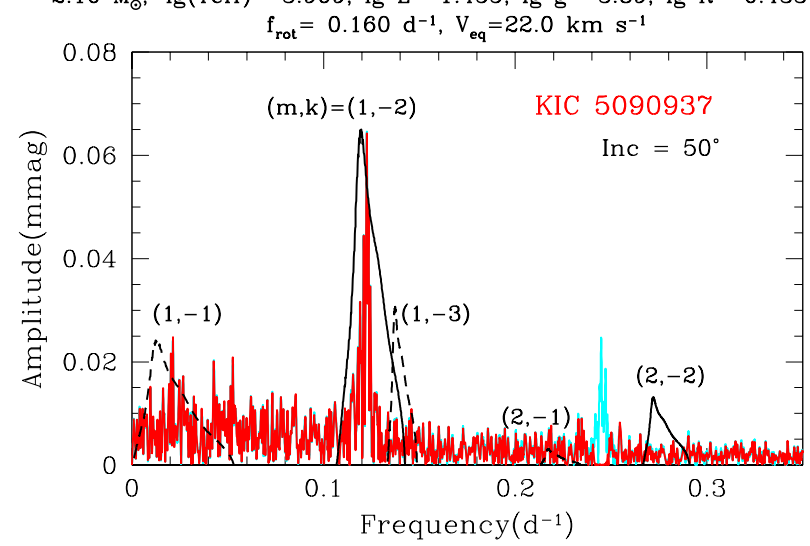

Figure A3. FTs for KIC 5090937. The cyan line denotes harmonics and combination frequencies of the relatively high-amplitude $(>0.03 \mathrm{mmag}$ ) peaks around $0.12 \mathrm{~d}^{-1}$.

$\mathrm{r}$ modes of $(m, k)=(1,-2)$ at a rotation frequency of $0.160 \mathrm{~d}^{-1}$. The determined rotation frequency corresponds to $P_{\text {rot }}=6.25 \mathrm{~d}$, which is comparable to $P_{\mathrm{ps} \text {-rot }}$.

\section{A4 KIC 5877364 (HD 183613)}

KIC 5877364 has a long orbital period of $89.649 \mathrm{~d}$ (KEBC) $\left(f_{\text {orb }}=0.0112 \mathrm{~d}^{-1}\right)$ with a very large orbital eccentricity of $e=$ $0.8875 \pm 0.031$ (Shporer et al. 2016), which yields the pseudosynchronous period $P_{\text {ps-rot }}=2.99 \pm 0.13$ d. Fig. A4 shows FTs for KIC 5877364. After removing dense orbital peaks (upper panel), a clear group of frequencies at $\sim 0.2-0.3 \mathrm{~d}^{-1}$ (red line) is apparent (lower panel), to which we fit the frequency range of $(m, k)=(1$, $-2) \mathrm{r}$ modes in a $1.80-\mathrm{M}_{\odot}$ model at a rotation frequency of $0.30 \mathrm{~d}^{-1}$ $\left(P_{\text {rot }}=3.33 \mathrm{~d}\right)$. The rotation period is consistent with the pseudosynchronous rotation period. Because of the very long orbital period, many orbital harmonic frequencies lie within $r$-mode frequency ranges so that the amplitudes of these $\mathrm{r}$ modes might be enhanced by tidal effects.

\section{A5 KIC 5960989}

The orbital period of the heartbeat binary KIC 5960989 is $50.722 \mathrm{~d}$ (KEBC), while the eccentricity $0.810 \pm 0.016$ is adopted in Table 1 by avaraging the results of Shporer et al. (2016) and Dimitrov et al. (2017). Using these orbital parameters in equation (3), we obtain $P_{\text {ps-rot }}=3.80 \pm 0.50$ d. Fig. A5 (lower panel) shows an FT for KIC 5960989. Also shown are visibility distributions of $\mathrm{r}$ modes in a $1.7-\mathrm{M}_{\odot}$ model (see Table 1) at a rotation frequency, $f_{\text {rot }}=0.22 \mathrm{~d}^{-1}$, which is chosen for the frequency range of $(m, k)=(1,-2) \mathrm{r}$ modes to be consistent with the frequency group around $0.16 \mathrm{~d}^{-1}$. The rotation frequency corresponds to the period $4.54 \mathrm{~d}$, which is comparable to the pseudo-synchronous rotation period.
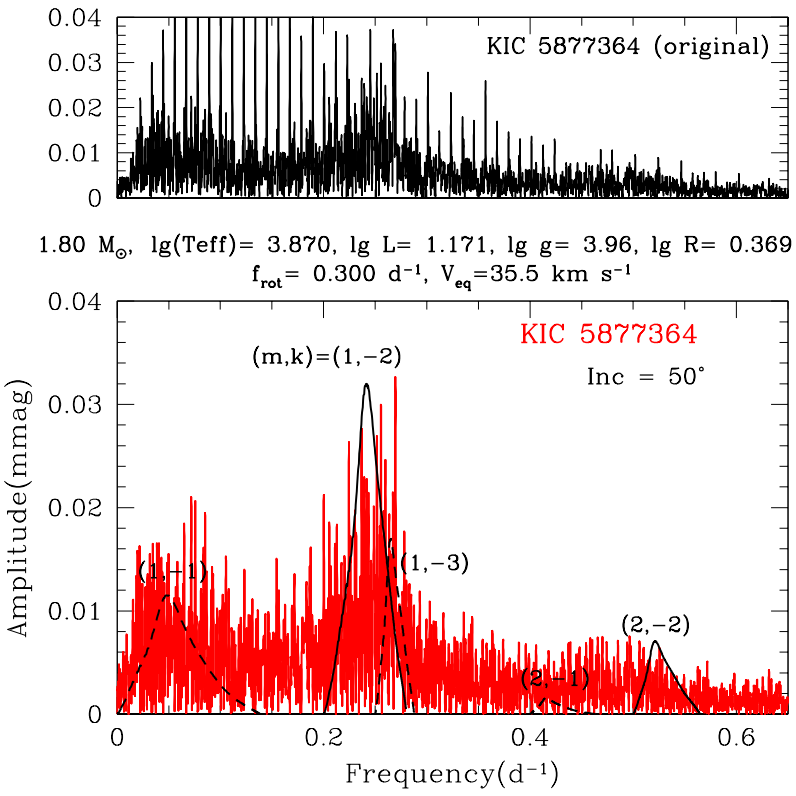

Figure A4. FTs for KIC 5877364 and visibility distributions of $\mathrm{r}$ modes in a $1.80-\mathrm{M}_{\odot}$ model at $f_{\text {rot }}=0.30 \mathrm{~d}^{-1}$.

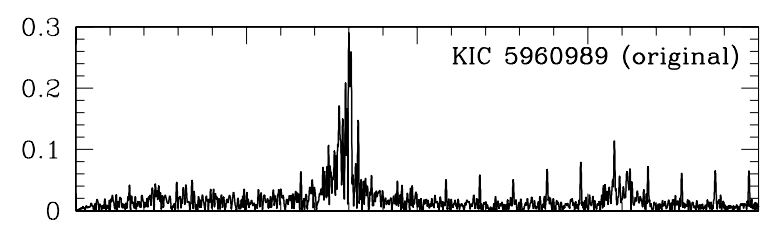

$1.70 \mathrm{M}_{\odot}, \lg (\mathrm{Teff})=3.812, \lg \mathrm{L}=1.086, \lg \mathrm{g}=3.78, \lg \mathrm{R}=0.443$

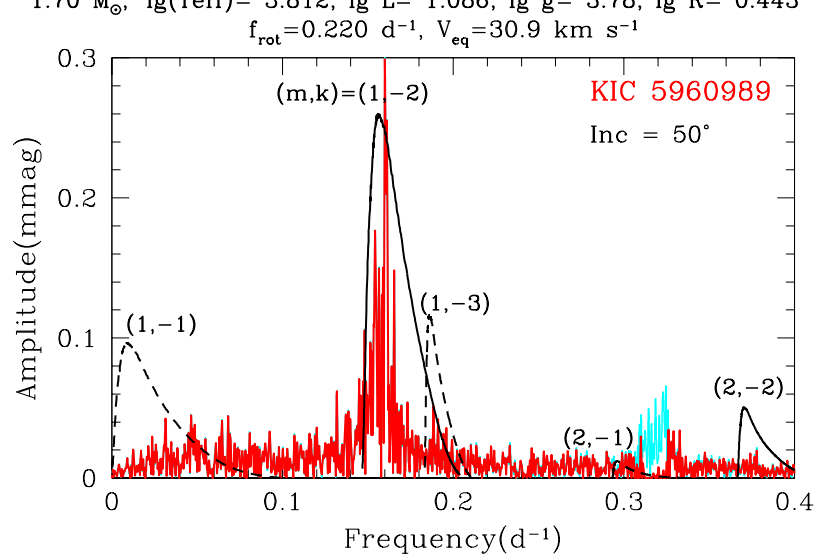

Figure A5. FTs for KIC 5960989 and visibility distributions of $\mathrm{r}$ modes in a $1.70-\mathrm{M}_{\odot}$ model at a rotation frequency of $0.22 \mathrm{~d}^{-1}$. The cyan line denotes frequencies identified as harmonics and combination frequencies of the frequencies whose amplitudes are larger than $0.1 \mathrm{mmag}$. The case of inclination $50^{\circ}$ is shown because Dimitrov et al. (2017) obtained an orbital inclination of $51.0 \pm 0.3$.

\section{A6 KIC 6117415}

KIC 6117415 is a heartbeat system with an orbital period of $19.742 \mathrm{~d}$ (KEBC) $\left(f_{\text {orb }}=0.0507 \mathrm{~d}^{-1}\right)$. Fig. A6 shows FTs for KIC 6117415 in a similar format to that of Fig. 1. R-mode visibility distributions are shown for a $1.45-\mathrm{M}_{\odot}$ main-sequence model at a rotation frequency of $0.43 \mathrm{~d}^{-1}$ assuming rotational inclination angles of $70^{\circ}$ and $30^{\circ}$. 


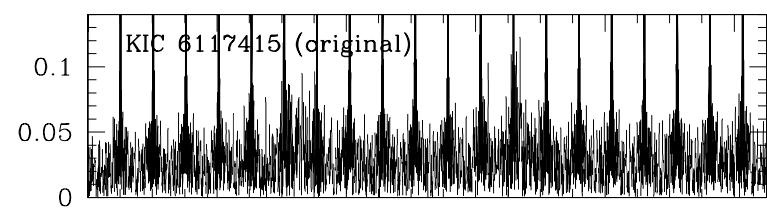

$1.45 \mathrm{M}_{\odot}, \lg ($ Teff $)=3.819, \lg \mathrm{L}=0.777, \lg \mathrm{g}=4.05, \lg \mathrm{R}=0.274$

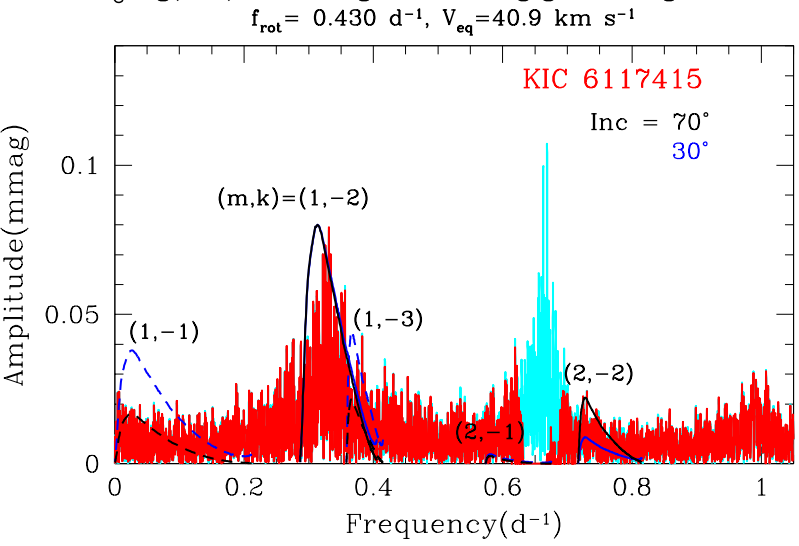

Figure A6. FTs for KIC 6117415 and theoretical visibility distributions of a model at a rotation frequency of $0.43 \mathrm{~d}^{-1}$. The cyan line shows harmonics and combination frequencies of high-amplitude peaks ( $\gtrsim 0.06 \mathrm{mmag}$ ) in the group at $\sim 0.3 \mathrm{~d}^{-1}$. The visibility of $\mathrm{r}$ modes is normalized as $0.08 \mathrm{mmag}$ at the maximum $\left(\sim 0.3 \mathrm{~d}^{-1}\right)$ of $(m, k)=(1,-2) \mathrm{r}$ modes. It can be seen that some of the combination frequencies have higher amplitudes than the parent peaks. Kurtz et al. (2015, section 2.3) discussed this and showed theoretically how it occurs.

The frequency group at $0.3-0.35 \mathrm{~d}^{-1}$ is well fitted with $(m, k)=$ $(1,-2) \mathrm{r}$ modes at $f_{\text {rot }}=0.43 \mathrm{~d}^{-1}$.

Cheng et al. (2020) obtained an orbital eccentricity of 0.734 for KIC 6117415. Using that value of eccentricity and the orbital period $19.742 \mathrm{~d}$ in equation (3), we obtain $P_{\mathrm{ps} \text {-rot }}=2.502 \mathrm{~d}$, which is comparable to the rotation period $2.33 \mathrm{~d}$ that corresponds to the assumed rotation frequency $f_{\text {rot }}=0.43 \mathrm{~d}^{-1}$. Cheng et al. (2020) also obtained an orbital inclination of 83.2 and a projected rotation velocity of $v \sin i=19-20 \mathrm{~km} \mathrm{~s}^{-1}$ for KIC 6117415 . Since our model predicts an equatorial rotation velocity of $41 \mathrm{~km} \mathrm{~s}^{-1}$, the inclination of the rotational axis should be about $30^{\circ}$, which is inclined to the orbital axis by about $50^{\circ}$. (Such a misalignment is also found in KIC 8164262 as discussed in Section 3.1.) For the rotational inclination of $30^{\circ}$ (blue line in Fig. A6), amplitudes of $(m, k)=(1,-1) \mathrm{r}$ modes near to $0 \mathrm{~d}^{-1}$ are predicted to be larger (under the energy equipartition assumption) than observed, which could indicate the antisymmetric $r$ modes to be less strongly excited. Cheng et al. (2020) also analysed another two heartbeat stars, KIC 11494130 and KIC 5790807. However, no clear r-mode features are found in the FTs of those binaries.

\section{A7 KIC 8027591}

The heartbeat binary KIC 8027591 has an orbital period $24.274 \mathrm{~d}$ (KEBC), and Shporer et al. (2016) obtained an orbital eccentricity of $0.586 \pm 0.008$. From these parameters equation (3) yields $P_{\mathrm{ps}-\mathrm{rot}}=$ $6.30 \pm 0.20$ d. Fig. A7 (lower panel) shows an FT for KIC 8027591 and predicted visibility distributions for $\mathrm{r}$ modes in a $1.50-\mathrm{M}_{\odot}$ model at a rotation frequency of $0.16 \mathrm{~d}^{-1}\left(P_{\text {rot }}=6.25 \mathrm{~d}\right)$, which is chosen to make the frequency range of $(m, k)=(1,-2) \mathrm{r}$ modes consistent

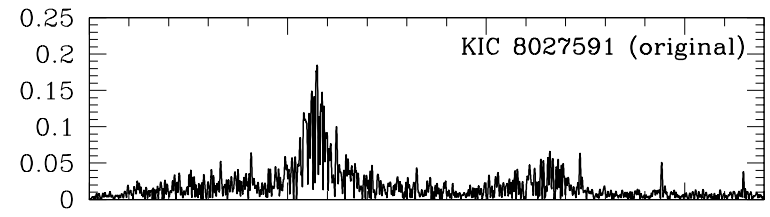

$1.50 \mathrm{M}_{\odot}, \lg ($ Teff $)=3.800, \lg \mathrm{L}=0.859, \lg \mathrm{g}=3.91, \lg \mathrm{R}=0.354$

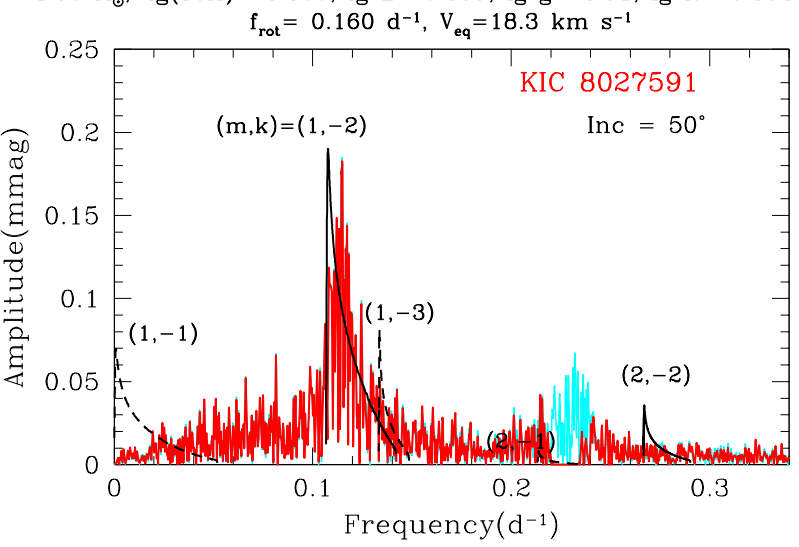

Figure A7. FTs for KIC 8027591. The cyan line in the lower panel denotes harmonics and combination frequencies resulting from frequencies in the main group with amplitudes larger than 0.1 mmag.

with the observed frequency group around $0.12 \mathrm{~d}^{-1}$. The obtained rotation period agrees with the $P_{\text {ps-rot }}$.

\section{A8 KIC 8719324}

KIC 8719324 is a heartbeat binary with an orbital period of $10.233 \mathrm{~d}$ (KEBC) and an eccentricity of $0.5998 \pm 0.0001$ (Guo et al. 2020), ${ }^{6}$ which yield $P_{\text {ps-rot }}=2.512 \mathrm{~d}$ from equation (3). Fig. A8 shows FTs for KIC 8719324; as in the other figures, the top panel shows the FT obtained from the original data including orbital harmonics, which are removed in the lower panel.

Zimmerman et al. (2017) identified the peaks at $\sim 0.323$ and $\sim 0.646 \mathrm{~d}^{-1}$ as rotational modulations, hence obtained the rotation period of KIC 8719324 to be $3.1 \mathrm{~d}$. Since the above-mentioned features are not single peaks but consist of dense groups of peaks, we fit the group of peaks at $\sim 0.323 \mathrm{~d}^{-1}$ with $(m, k)=(1,-2)$ r modes in a $1.80-\mathrm{M}_{\odot}$ model at a rotation frequency of $0.38 \mathrm{~d}^{-1}\left(P_{\text {rot }}=2.63 \mathrm{~d}\right)$. Our rotation period, $2.63 \mathrm{~d}$, is similar to the pseudo-synchronous rotation $2.51 \mathrm{~d}$.

\section{A9 KIC 9790355}

The orbit of the heartbeat binary KIC 9790355 has a period of $14.566 \mathrm{~d}(\mathrm{KEBC})$ and an eccentricity of $0.513 \pm 0.007$ (Thompson et al. 2012). From these parameters equation (3) gives a pseudosynchronous period $P_{\text {ps-rot }}=4.96 \pm 0.12 \mathrm{~d}$. Fig. A9 shows FTs for KIC 9790355 with $\mathrm{r}$-mode visibility distributions for a $1.80-\mathrm{M}_{\odot}$ model in a similar format to Fig. 1. In order to fit the frequency group at $\sim 0.16$ with the frequency range of $(m, k)=(1,-2) \mathrm{r}$ modes, a

${ }^{6}$ The eccentricity was obtained by fixing $P_{\text {orb }}$ at the KEBC value, while Smullen \& Kobulnicky (2015) obtained $e=0.64 \pm 0.05$ and $P_{\text {orb }}=$ $10.235 \pm 0.005 \mathrm{~d}$. We have adopted, in Table 1 , the eccentricity based on the fixed $P_{\text {orb }}$. 

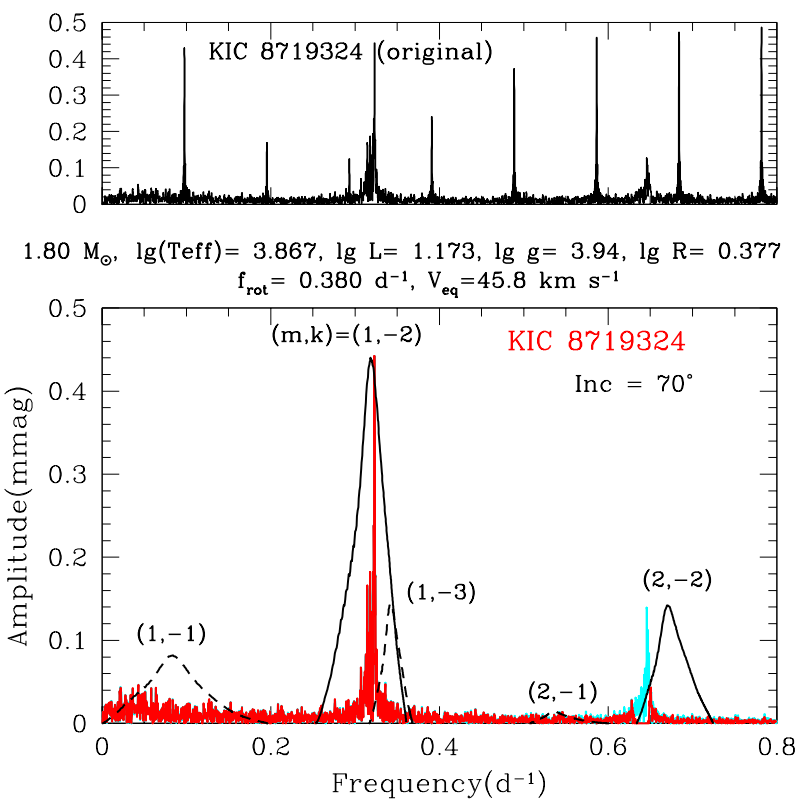

Figure A8. FTs for KIC 8719324; the upper panel includes orbital harmonics, which are removed in the lower panel. The cyan line denotes harmonics and combination frequencies of the highest peaks $(\gtrsim 0.1 \mathrm{mmag})$ of the frequency group at $0.32 \mathrm{~d}^{-1}$. Guo et al. (2020) obtained an orbital inclination of $73.54 \pm 0.06$. They also found tidally excited pulsations at $\sim 2.5$ and $\sim 3 \mathrm{~d}^{-1}$, which are out of the range of this diagram.

rotation frequency of $0.22 \mathrm{~d}^{-1}$ is adopted. The corresponding rotation period, $4.76 \mathrm{~d}$, is similar to $P_{\mathrm{ps}-\mathrm{rot}}$ of KIC 9790355.

\section{A10 KIC 10334122}

KIC 10334122 is a relatively long period $\left(P_{\text {orb }}=37.953 \mathrm{~d}\right.$; KEBC) heartbeat star. The orbital eccentricity was determined by Shporer et al. (2016) to be $0.53 \pm 0.06$. Using the orbital period and eccentricity in equation ( 3 ) yields $P_{\mathrm{ps}-\text { rot }}=12.0 \pm 2.6 \mathrm{~d}$, which is considerably longer than the rotation period $5.88 \mathrm{~d}$ obtained by fitting the frequency group at $\sim 0.12 \mathrm{~d}^{-1}$ by the $(m, k)=(1,-2) \mathrm{r}$ modes (Fig. A10). Among our sample heartbeat stars, this is the only case where $P_{\text {ps-rot }} \gg P_{\text {rot }}$.

\section{A11 KIC 11071278}

KIC 11071278 is a relatively cool $\left(T_{\text {eff }}=5900 \pm 120 \mathrm{~K}\right.$; Berger et al. 2020) heartbeat binary with an orbital period of $55.885 \mathrm{~d}$ (KEBC). Shporer et al. (2016) estimated an orbital eccentricity of 0.755 . With these parameters equation (3) gives a pseudo-synchronous rotation period $P_{\mathrm{ps} \text {-rot }}=6.23 \mathrm{~d}$. Fig. A11 (lower panel) shows an FT for KIC 11071278. Regarding all frequencies in the hump at $\sim 0.22 \mathrm{~d}^{-1}$ as harmonics and combination frequencies, we fit the main hump at $\sim 0.11 \mathrm{~d}^{-1}$ with $(m, k)=(1,-2)$ r modes, assuming a rotation frequency of $0.15 \mathrm{~d}^{-1}\left(P_{\text {rot }}=6.67 \mathrm{~d}\right)$. The assumed rotation frequency is consistent with $P_{\mathrm{ps}-\text { rot }}$ for KIC 11071278 .

\section{A12 KIC 11403032}

The orbital period of KIC 11403032 is $7.6316 \mathrm{~d}$ (KEBC), while the eccentricity $e=0.288 \pm 0.013$ was obtained by Shporer et al. (2016). Equation (3) with these parameters gives $P_{\mathrm{ps}-\text { rot }}=5.05 \pm 0.22 \mathrm{~d}$. The

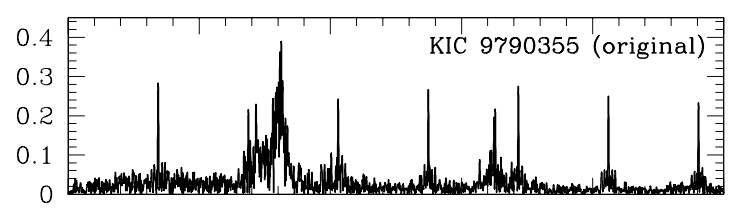

$1.70 \mathrm{M}_{\odot}, \lg ($ Teff $)=3.812, \lg \mathrm{L}=1.086, \lg \mathrm{g}=3.78, \lg \mathrm{R}=0.443$ $\mathrm{f}_{\text {rot }}=0.220 \mathrm{~d}^{-1}, \mathrm{~V}_{\text {eq }}=30.9 \mathrm{~km} \mathrm{~s}^{-1}$

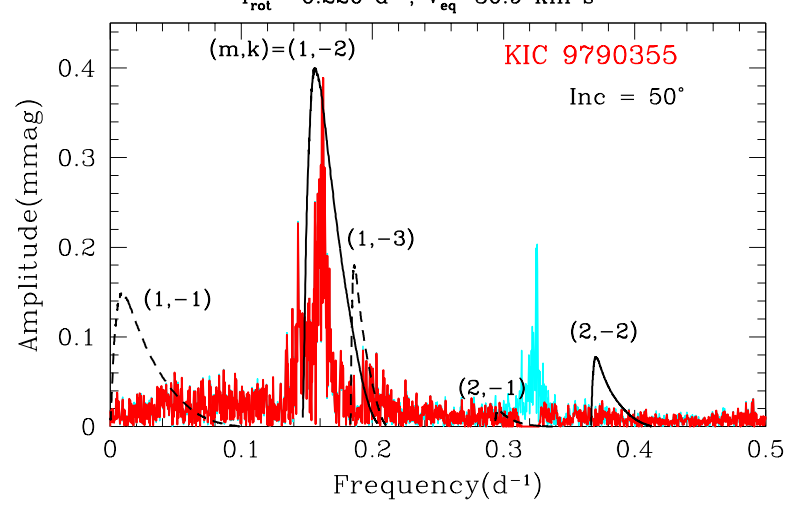

Figure A9. FTs for KIC 9790355. Harmonics and combination frequencies of high-amplitude ( $>0.2 \mathrm{mmag}$ ) peaks at $\sim 0.16 \mathrm{~d}^{-1}$ are shown by the cyan line. R-mode visibilities for an inclination of $50^{\circ}$ are shown because Thompson et al. (2012) obtained an orbital inclination of $46.3 \pm 0.7$ for this star.

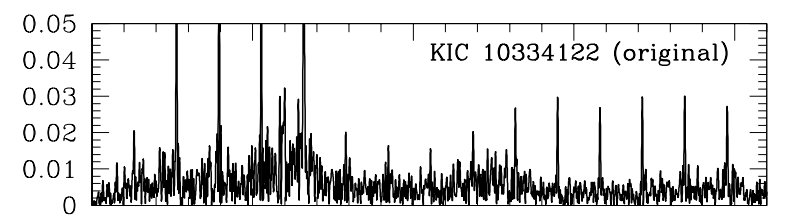

$1.65 \mathrm{M}_{\odot}, \lg ($ Teff $)=3.809, \lg \mathrm{L}=1.032, \lg \mathrm{g}=3.81, \lg \mathrm{R}=0.422$

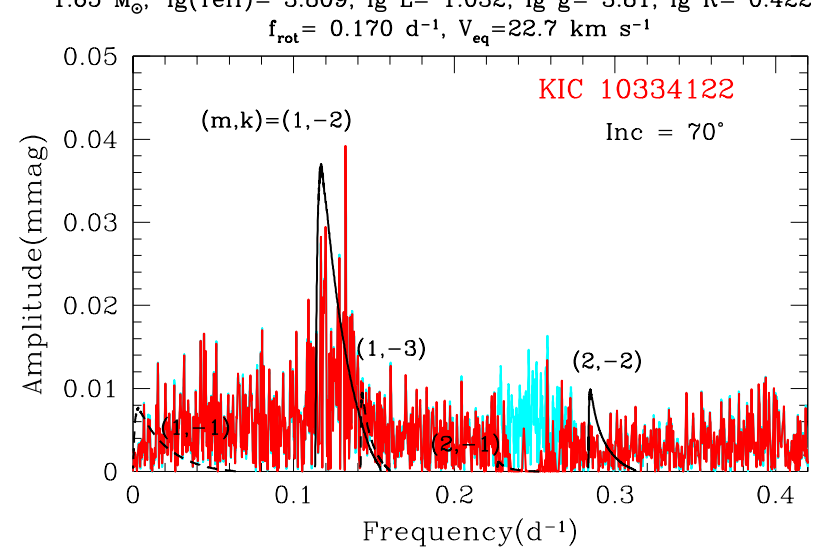

Figure A10. FTs for KIC 10334122. The cyan line indicates harmonics and combination frequencies of 10 highest amplitude $(>0.02 \mathrm{mmag})$ peaks in the frequency group around $0.12 \mathrm{~d}^{-1}$, which is fitted with $(m, k)=(1,-2)$ at a rotation frequency of $0.17 \mathrm{~d}^{-1}$.

rotation period obtained by our $r$ mode fitting (Fig. A12) is $10.5 \mathrm{~d}$ ( $f_{\text {rot }}=0.095 \mathrm{~d}^{-1}$ ), which is longer than $P_{\mathrm{ps} \text {-rot }}$ by about a factor of 2. The rotation of KIC 11403032 is the slowest among our sample. Zimmerman et al. (2017) obtained a still longer rotation period of $14.89 \pm 0.22 \mathrm{~d}$ for KIC 11403032 assuming the frequency group at $\sim 0.07 \mathrm{~d}^{-1}$ in the FT to be caused by surface spots. 


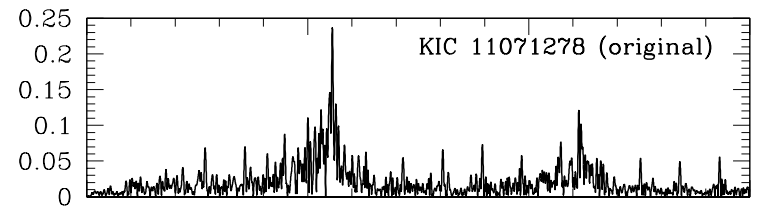

$1.60 \mathrm{M}_{\odot}, \lg ($ Teff $)=3.803, \lg \mathrm{L}=0.980, \lg \mathrm{g}=3.83, \lg \mathrm{R}=0.407$

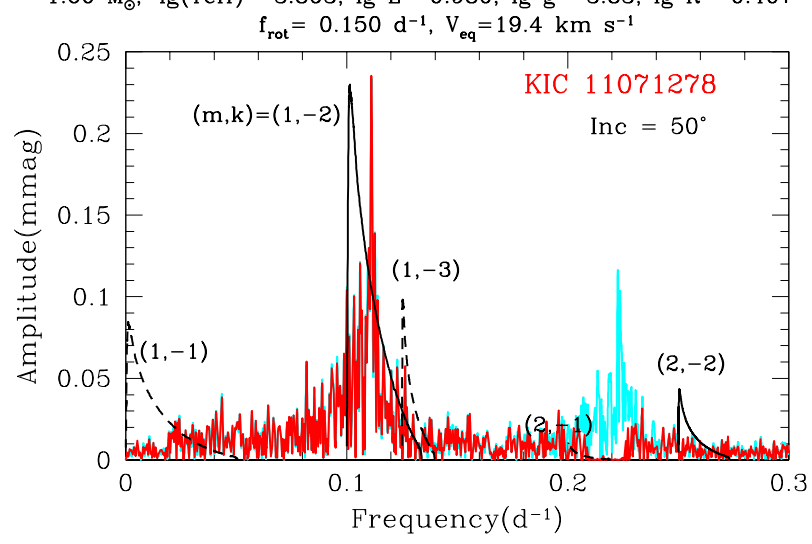

Figure A11. FTs for KIC 11071278. The cyan line indicates harmonics and combination frequencies of the highest amplitude peaks $(>0.1 \mathrm{mmag})$ around $0.11 \mathrm{~d}^{-1}$.

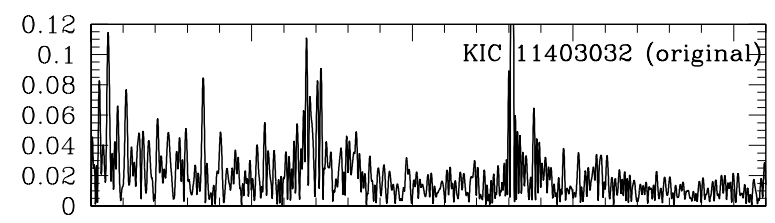

$1.80 \mathrm{M}_{\odot}, \lg ($ Teff $)=3.822, \lg \mathrm{L}=1.193, \lg \mathrm{g}=3.74, \lg \mathrm{R}=0.476$

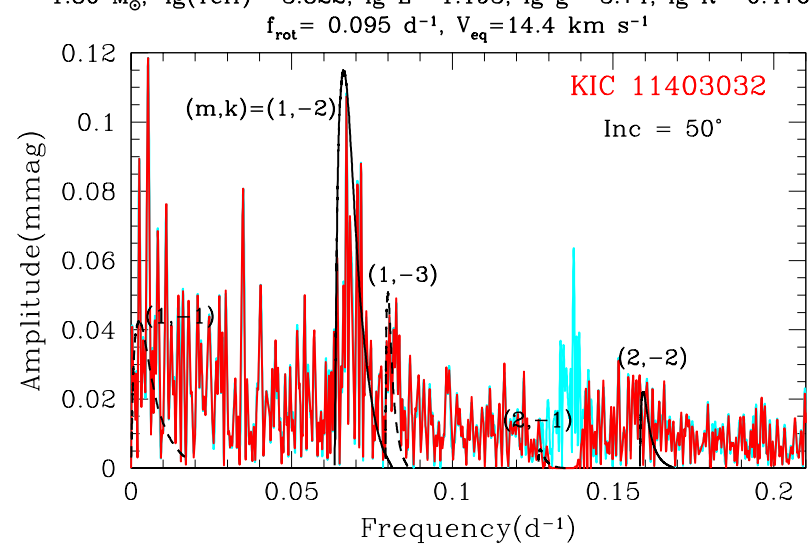

Figure A12. FTs for KIC 11403032 obtained from a KEBC (Kirk et al. 2016) de-trended light curve. The upper panel shows an FT from the original data including the orbital frequency at $0.131 \mathrm{~d}^{-1}$. The orbital frequency is removed in the lower panel. The cyan line denotes harmonics and combination frequencies of high-amplitude ( $>0.05 \mathrm{mmag}$ ) peaks around $0.07 \mathrm{~d}^{-1}$. The group of peaks around $0.07 \mathrm{~d}^{-1}$ is fitted with $(m, k)=(1,-2) \mathrm{r}$ modes in a $1.8-\mathrm{M}_{\odot}$ model at a rotation frequency of $0.095 \mathrm{~d}^{-1}$. A narrow group at $\sim 0.08 \mathrm{~d}^{-1}$ agrees with with odd $(m, k)=(1,-3) \mathrm{r}$ modes. Furthermore, the relatively small amplitude group at $\sim 0.16 \mathrm{~d}^{-1}$ likely corresponds to $(m, k)=$ $(2,-2) \mathrm{r}$ modes.

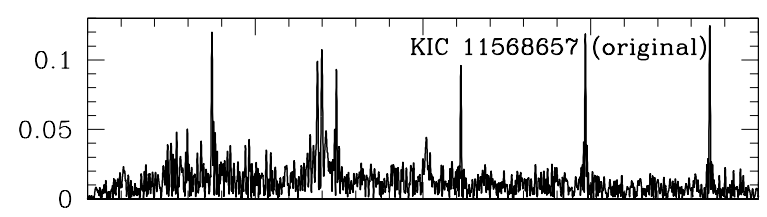

$1.60 \mathrm{M}_{\odot}, \lg ($ Teff $)=3.809, \lg \mathrm{L}=0.974, \lg \mathrm{g}=3.86, \lg \mathrm{R}=0.393$

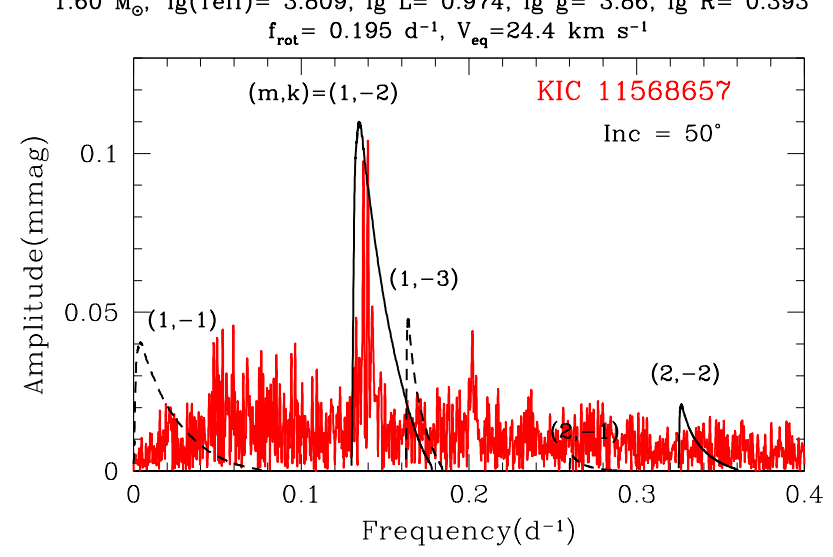

Figure A13. FTs for the heartbeat star KIC 11568657. The frequency group at $\sim 0.14 \mathrm{~d}^{-1}$ is fitted with $(m, k)=(1,-2) \mathrm{r}$ modes in a $1.6-\mathrm{M}_{\odot}$ model with a rotation frequency of $0.195 \mathrm{~d}^{-1}$. The amplitude peak at $0.2 \mathrm{~d}^{-1}$ is possibly caused by oscillatory convective modes in the convective core (Lee \& Saio 2020; Lee 2021), or a spot on the surface.
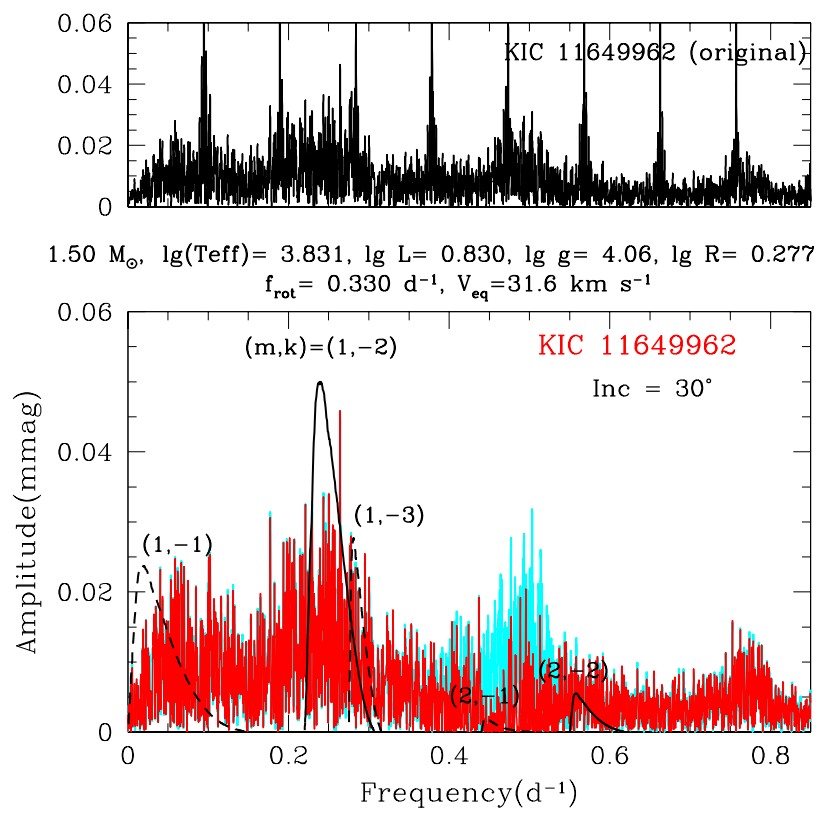

Figure A14. FTs for KIC 11649962. The cyan line indicates harmonics and combination frequencies of 15 highest amplitude $(>0.027 \mathrm{mmag}$ ) frequencies around $0.25 \mathrm{~d}^{-1}$. The frequency group at $\sim 0.25 \mathrm{~d}^{-1}$ is fitted with the visibility curve of $(m, k)=(1,-2) \mathrm{r}$ modes in the $1.5-\mathrm{M}_{\odot}$ model at a rotation frequency of $0.33 d^{-1}$. 


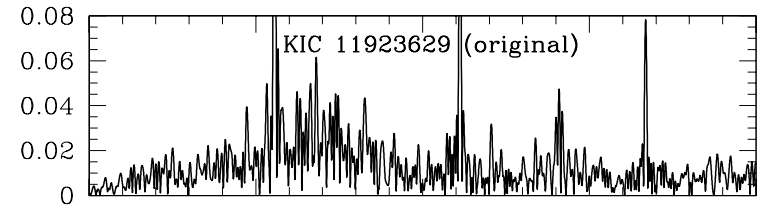

$1.50 \mathrm{M}_{\odot}, \lg (\mathrm{Teff})=3.801, \lg \mathrm{L}=0.858, \lg \mathrm{g}=3.91, \lg \mathrm{R}=0.351$

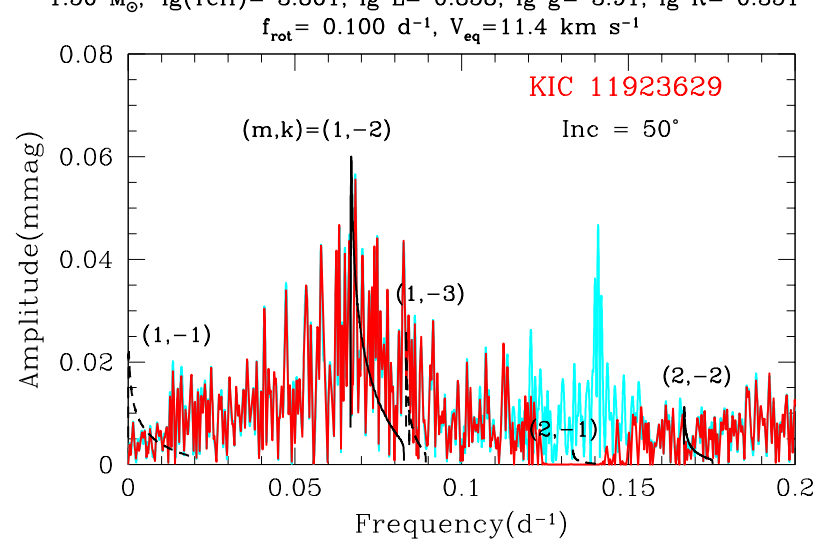

Figure A15. FTs for KIC 11923629. The cyan line denotes frequencies corresponding to harmonics and combination frequencies among highest amplitude (>0.04 mmag) frequencies around $0.07 \mathrm{~d}^{-1}$. The frequency group around $0.07 \mathrm{~d}^{-1}$ is fitted with $(m, k)=(1,-2) \mathrm{r}$ modes in a $1.50-\mathrm{M}_{\odot}$ model at a rotation frequency of $0.10 \pm 0.01 \mathrm{~d}^{-1}$.

\section{A13 KIC 11568657}

The heartbeat star KIC 11568657 has an orbital period of $13.476 \mathrm{~d}$ (KEBC). Thompson et al. (2012) obtained the orbital eccentricity $e=$ $0.565 \pm 0.002$ and inclination $i=48.2 \pm 0$. 1 . From the orbital period and the eccentricity, equation (3) yields $P_{\text {ps-rot }}=3.798 \pm 0.029 \mathrm{~d}$, which is somewhat shorter than the rotation period $5.13 \mathrm{~d}$ obtained by the fitting the frequency group at $\sim 0.14 \mathrm{~d}^{-1}$ with $(m, k)=(1,-2)$ $r$ modes (Fig. A13).

\section{A14 KIC 11649962}

The heartbeat star KIC 11649962 has an orbital period of $10.563 \mathrm{~d}$ (KEBC). Shporer et al. (2016) obtained the eccentricity $e=$ $0.5206 \pm 0.0035$. Using the orbital period and the eccentricity in equation (3), we obtain $P_{\text {ps-rot }}=3.505 \pm 0.043 \mathrm{~d}$, which is comparable to the rotation period $3.03 \mathrm{~d}$ obtained by fitting the frequency group at $\sim 0.25 \mathrm{~d}^{-1}$ with the $(m, k)=(1,-2) \mathrm{r}$ modes (Fig. A14).

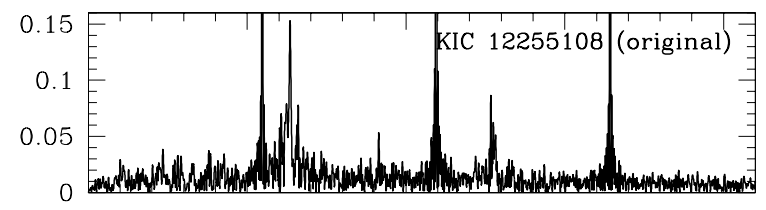

$1.90 \mathrm{M}_{\odot}, \lg ($ Teff $)=3.901, \lg \mathrm{L}=1.253, \lg \mathrm{g}=4.02, \lg \mathrm{R}=0.347$

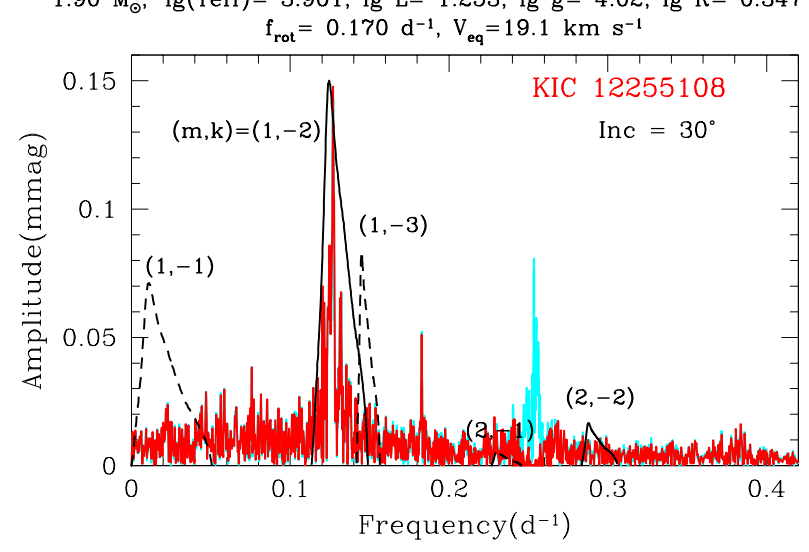

Figure A16. FTs for KIC 12255108. The cyan line denotes harmonics and combination frequencies of high-amplitude peaks (>0.06 mmag) around $0.12 \mathrm{~d}^{-1}$.

\section{A15 KIC 11923629}

The heartbeat star KIC 11923629 has an orbital period of $17.973 \mathrm{~d}$ (KEBC). Shporer et al. (2016) obtained the eccentricity $e=$ $0.363 \pm 0.006$. From the orbital period and eccentricity, we obtain $P_{\text {ps-rot }}=9.77 \pm 0.16 \mathrm{~d}$, which is comparable to the rotation period $10.0 \mathrm{~d}$ obtained by fitting with r-mode frequency range (Fig. A15), while (Zimmerman et al. 2017) obtained a slightly longer rotation period of $15.79 \pm 0.15 \mathrm{~d}$ by assuming the frequency group at $\sim 0.07 \mathrm{~d}^{-1}$ to be generated by surface spots.

\section{A16 KIC 12255108}

The heartbeat binary KIC 12255108 has an orbital period $P_{\text {orb }}=$ $9.1315 \mathrm{~d}$ (KEBC), and the orbital eccentricity $e=0.296$ obtained by Shporer et al. (2016). Then, equation (3) gives the pseudosynchronous period $P_{\text {ps-rot }}=5.92 \mathrm{~d}$. Fig. A16 (lower panel) shows an FT for KIC 12255108 fitted with the visibility of $\mathrm{r}$ modes at a rotation frequency of $0.17 \mathrm{~d}^{-1}\left(P_{\text {rot }}=5.88 \mathrm{~d}\right)$ for a $1.90-\mathrm{M}_{\odot}$ main-sequence model. The obtained rotation period is similar to the pseudo-synchronous rotation period.

This paper has been typeset from a $\mathrm{T}_{\mathrm{E}} \mathrm{X} / \mathrm{L} \mathrm{A} \mathrm{E} \mathrm{X}$ file prepared by the author. 